\title{
Super Loop Groups, Hamiltonian Actions and Super Virasoro Algebras ${ }^{\star}$
}

\author{
J. Harnad ${ }^{1}$ and B. A. Kupershmidt ${ }^{2}$ \\ ${ }^{1}$ Department of Mathematics, Concordia University, and Centre de Recherches Mathématiques, \\ Université de Montréal, C.P. 6128-A, Montréal, Qué., Canada, H3C 3J7 \\ ${ }^{2}$ The University of Tennessee Space Institute, Tullahoma, TN 37388, USA
}

\begin{abstract}
The quotient $\widetilde{L G} / G$ of a super loop group $\widetilde{L G}$ by the subgroup of constant loops is given a supersymplectic structure and identified through a moment map embedding $\widetilde{J}^{L \wedge}: \widetilde{L G} / G \rightarrow \widetilde{L g}^{\wedge} *$ with a coadjoint orbit of the centrally extended super loop algebra $\widetilde{L g^{\wedge}}$. The algebra $\widetilde{\operatorname{diff}}^{c} S^{1}$ of superconformal vector fields on the circle is shown to have a natural representation as Hamiltonian vector fields on $\widetilde{L G} / G$ generated by an equivariant moment map $\widetilde{J}: \widetilde{L G} / G \rightarrow{\widetilde{\operatorname{diff}^{c}}}^{c} S^{1 *}$. This map is obtained by composition of $\widetilde{J}^{L \wedge}$ with a super Poisson map $\widetilde{J}^{\varphi}: \widetilde{L g}^{\wedge} \rightarrow \widetilde{\operatorname{diff}}^{c} S^{1 *}$ defining a supersymmetric extension of the classical Sugawara formula. Upon quantization, this yields the corresponding formula of Kac and Todorov on unitary highest weight representations. For any homomorphism $\rho: u(1) \rightarrow G$, an associated "twisted" moment map $\widetilde{J}^{\rho}: \widetilde{L G} / G \rightarrow \widetilde{\operatorname{diff}}_{\mathbb{C}} S^{1 *}$ is also derived, generating a super Poisson bracket realization of a super Virasoro subalgebra $\widetilde{\mathrm{Vir}}$ of the semi-direct sum $\widetilde{\mathrm{diff}}_{\mathbb{C}}^{c} S^{1} \ltimes \widetilde{\mathrm{Lg}}^{\wedge}$. The corresponding super Poisson map $\widetilde{J}^{\mathscr{\rho} \rho}: \widetilde{L g}^{\wedge} * \rightarrow \widetilde{V i r}^{*}$ is interpreted as a nonabelian generalization of the super Miura map and applied to two super $\mathrm{KdV}$ hierarchies to derive corresponding integrable generalized super MKdV hierarchies in $\widetilde{L g^{\wedge *}}$.
\end{abstract}

\section{Introduction}

In the Hamiltonian framework, the classical Sugawara formula [S] for a $1+1$ dimensional field theory may be viewed as a Poisson map $J^{\mathscr{S}}: L \mathfrak{g}^{\wedge} \rightarrow \operatorname{diff} S^{1 *}$ from the dual of the centrally extended loop algebra $\mathrm{Lg}^{\wedge}$ to the dual of the algebra $\operatorname{diff} S^{1}$ of vector fields on the circle. The Poisson space $L \mathfrak{g}^{\wedge}$ enters as a "universal" phase space into which conformally invariant models may be mapped via their currents. The latter are interpreted in the Hamiltonian framework as moment maps generating the action of the loop group $L G$ in terms of Hamiltonian flows. The

\footnotetext{
^ Research supported in part by the Natural Sciences and Engineering Research Council of Canada and the National Science Foundation (USA)
} 
coadjoint orbit $\mathcal{O}_{(0,1)} \subset L \mathfrak{g}^{\wedge *}$ (where $\left.(0,1) \in L \mathfrak{g}^{*} \oplus \mathbb{R} \equiv L \mathfrak{g}^{\wedge}\right)$ figures as a reduced phase space underlying the Wess-Zumino-Witten model ([W], $[\mathrm{H}])$ and also plays an important rôle in the representation theory of loop groups [PS]. This orbit may be identified with the coset space $L G / G$ (where $G$ is understood as the subgroup of constant loops) or, equivalently, with the group $\Omega G$ of based loops, an infinite dimensional Kähler manifold [PS]. The embedding $J^{L}: \Omega G \subseteq L \mathfrak{g}^{\wedge}{ }^{*}$ defines a moment map that generates the natural left $L G$-action in terms of Hamiltonian flows.

The group Diff $\mathscr{S}^{1}$ acts naturally on $L G / G$ by reparametrization of loops and this action is also Hamiltonian, with equivariant moment map $J: L G / G \rightarrow \operatorname{diff} S^{1 *}$ defined by composition $J=J^{\mathscr{S}} \circ J^{L}$. This factorization underlies the classical derivation of the Sugawara formula for Hamiltonian models having $L G / G$ as phase space $[\mathrm{H}]$. It may also be used as a starting point for quantization and for development of the representation theory of the semi-direct sum $\operatorname{diff} S^{1} \ltimes L \mathfrak{g}^{\wedge}$.

For applications to 2-dimensional super-conformal field theories, super-strings and the associated representation theory of super-algebras, it is important to extend the Hamiltonian framework to super loop groups $\widetilde{L G}$, the algebra $\widetilde{\operatorname{diff}}^{c} S^{1}$ of infinitesimal super-conformal transformations of the circle and their central extensions. In the abelian case, i.e. loops in a vector space or torus, this is easily done (see e.g. [GSW]), and gives rise upon quantization to the usual formulas for generators of the super Virasoro algebra expressed quadratically in terms of bosonic and fermionic creation and annihilation operators.

In the framework of representation theory, Kac and Todorov [KT] gave formulas of the Sugawara type allowing the construction of all unitary positive energy highest weight representations of the semi-direct product of the super Virasoro algebra with affine super Kac-Moody algebras. However the super Virasoro generators are not directly expressed in terms of the full Kac-Moody generators, but rather in terms of two parts, corresponding to the bosonic and fermionic contributions separately. The splitting is obtained by constructing the Hilbert space as a tensor product of a fermionic Fock space and a bosonic one that is an irreducible highest wieght module for the even part of the algebra. Thus, these formulas are specific to such representations rather than intrinsic to the algebras. The classical, Hamiltonian origins of such results are not a priori evident, and it is of some importance in linking the representation theory to 2-dimensional super conformal quantum field theory that they be derived through quantization upon some suitably defined phase space.

A further domain of applications for formulas of the Sugawara type occurs in the theory of classical completely integrable Hamiltonian systems. In particular, the Miura transformation relating solutions of the MKdV hierarchy to those of the KdV hierarchy may be viewed as an example of a "twisted" Sugawara formula in the abelian one-dimensional case, with loops replaced by maps of the real line. Infinite families of completely integrable systems on non-abelian loop or current algebras may also be obtained from analogous constructions [Ku1]. The extension of such considerations to super integrable systems of the super $\mathrm{KdV}$ and MKdV type is known ([Ku2], [Ma]). The corresponding extensions needed for the 
non-abelian case require a suitable development of the Hamiltonian approach to super loop groups.

It is our purpose in the present work to extend the Hamiltonian formulation of loop groups, loop algebras and $\operatorname{diff} S^{1}$, together with their moment map realizations, to their super-space counterparts. Thus, we are led to super loop group actions and representations, super trace-residue pairings, super symplectic forms and coadjoint orbits, super moment maps and a super version of the classical Sugawara formula.

In Sect. 2, the super loop group $\widetilde{L G}$ and algebra $\widetilde{L g}$ are introduced in terms of a supermanifold extension of $L G$, with extended structure sheaf $\tilde{\mathscr{F}}(\widetilde{L G})$ identified essentially with sections of the Grassmann algebra bundle $\Lambda(T L G)$. The super circle $S^{1}$ is defined with extended structure sheaf $\tilde{\mathscr{F}}\left(S^{1}\right)$ generated by adjoining an odd nilpotent element $\theta$ to the usual angular variable $\{\sigma \bmod 2 \pi\}$ on $S^{1}$. A superspace analogue of loops is used to characterize elements $\tilde{g} \in \overline{L G}$ as maps $\tilde{g}: \widetilde{S}^{1} \rightarrow G$ via the dual homomorphism $\tilde{g}^{*}: \mathscr{F}(G) \rightarrow \tilde{\mathscr{F}}\left(S^{1}\right)$ of structure sheaves. This leads to natural definitions for supergroup multiplication, adjoint and coadjoint actions. Group and algebra super cocycles are introduced to define the corresponding central extension $\mathbb{R} \rightarrow \widetilde{L g}^{\wedge} \rightarrow \widetilde{L g}$ together with the extended adjoint and coadjoint representations.

In Sect. 3 a supersymplectic form is defined on the coset space $\overparen{L G} / G$ in terms of the supercocycle. A non-equivariant moment map $\widetilde{J}^{L}: \widetilde{L G} / G \rightarrow \widetilde{L g}^{*}$ is derived for the Hamiltonian super group action of $\widetilde{L G}$ on $\widetilde{L G} / G$ (Theorem 3.3) and, using the corresponding cocycle, is used to define the extended equivariant map $\widetilde{J}^{L \wedge}: \widetilde{L G} / G \rightarrow \widetilde{L g}^{\wedge *}$. This map is injective, giving an identification of $\widetilde{L G} / G$ with the coadjoint orbit $\mathcal{O}_{(0, k)} \subset \widetilde{L g}^{\wedge *}$ as super symplectic manifolds.

In Sect. 4, the algebra $\widetilde{\operatorname{diff}}^{c} S^{1}$ of super conformal vector fields on $\tilde{S}^{1}$ is introduced, together with its representation in terms of Hamiltonian vector fields on $\widetilde{L G} / G$. The corresponding equivariant moment map $\widetilde{J}: \widetilde{L G} / G \rightarrow \widetilde{\operatorname{diff}^{c}} S^{1 *}$ is derived and shown, together with $\tilde{J}^{L \wedge}$, to define a Poisson bracket realization of the semi-direct sum $\widetilde{\operatorname{diff}}^{c} S^{1} \ltimes \widetilde{L g}^{\wedge}$. The map $\widetilde{J}$ factors through $\widetilde{\mathrm{Lg}^{\wedge} *}$ by composition of $\widetilde{J}^{L \wedge}$ with a super Poisson map $\widetilde{J}^{\mathscr{S}}: \widetilde{L g}^{\wedge} \rightarrow \widetilde{\operatorname{diff}}^{c} S^{1 *}$ (Eq. (4.32)). This gives the phase space form of the super Sugawara formula, expressing the $\widetilde{\operatorname{diff}}^{c} S^{1}$ generators in terms of those for the super loop algebra. Unlike the purely even case these expressions involve both quadratic and cubic terms. However, if the even $\widetilde{L g} \wedge$ generators are split into a sum $J_{B}^{L}+J_{F}^{L}$, where $J_{B}^{L}$ is defined on the even part of phase space and $J_{F}^{L}$ on the odd part, the $\widetilde{\operatorname{diff}}^{c} S^{1}$ generators are expressible quadratically in terms of these two terms, together with the odd $\widetilde{\mathrm{Lg}^{\wedge}}$ generators. In physical terms, the $\widetilde{\mathrm{Lg}}^{\wedge}$ moment map defines a super current, with even part an ordinary current consisting of the sum of a bosonic current $J_{B}^{L}$ and a fermionic one $J_{F}^{L}$ while the odd part is just a Fermi field in the adjoint representation of $\mathfrak{g}$.

In Sect. 5, the moment maps $\widetilde{J}^{L}$ and $\widetilde{J}$ are expressed in terms of their Fourier components and the Poisson bracket relations for $\widetilde{\operatorname{diff}}_{\mathbb{C}}^{c} S^{1} \ltimes \widetilde{\mathrm{Lg}^{\wedge}}$ together with the super Sugawara formula are given in terms of these components. In this classical framework, the super-Virasoro algebra has no center. However, in Sect. 6, a "twisting" of the $\widetilde{\operatorname{diff}^{c}} S^{1}$ action is introduced, associated to each 
homomorphism $\rho: U(1) \rightarrow G$, as in the even case [HK]. The resulting "twisted" $\widetilde{\operatorname{diff}}^{c} S^{1}$ representation is generated by a non-equivariant moment map and hence, in terms of Poisson brackets of generators, gives rise to a center associated to a nontrivial cocycle. In Sect. 7, these results are applied to determining hierarchies of superintegrable systems on $\widetilde{L g}^{\wedge}$, giving the super extensions of generalized MKdV systems. Finally, in Sect. 8, the quantization of the moment maps derived in Sects. 3-5 is shown to lead directly to results of the type found by Kac and Todorov $[\mathrm{KT}]$ in their construction of unitary highest weight representations.

\section{The Super Loop Group $\widetilde{L G}$ and Algebra $\widetilde{L g}$}

Let $L G$ denote the group of parametrized loops $\{g(\sigma)=g(\sigma+2 \pi) \in G\}$ in a Lie group $G$, with pointwise multiplication. The Lie algebra of $G$ is denoted $\mathfrak{g}$, and the associated loop algebra $L \mathrm{~g}$. The super loop group $\widetilde{L G}$ has an underlying supermanifold structure with $L G$ as reduced space and extended super structure sheaf $\tilde{\mathscr{F}}(\widetilde{L G})$ consisting essentially of sections of the Grassmann algebra bundle $\Lambda(T L G)$. Thus, $\widetilde{L G}$ is a "split" supermanifold [K]. Since the tangent bundle may be identified through left translation with the product $L G \times L \mathfrak{g}$, we may equally consider the super structure sheaf as $\mathscr{F}(L G) \otimes \Lambda(L g)$. Thus "points" in $\widetilde{L G}$ may be viewed as pairs $(g, \psi)$, where $g \in L G$ and $\psi \in L \mathfrak{g}$, the latter being regarded as an odd generator. Expressions of the form $g \psi$ or $\psi g$ should be interpreted as denoting sections of $T(L G)$ obtained from $\left.\psi \in T(L G)\right|_{\text {Id }}$ by left or right translation. We must also allow a slightly more general extension of $L G$, which for the most part will not be distinguished notationally, in which the odd generators $\psi(\sigma)$ do not necessarily satisfy periodic boundary conditions $\{\psi(\sigma)=\psi(\sigma+2 \pi)\}$, but possibly anti-periodic ones $\{\psi(\sigma)=-\psi(\sigma+2 \pi)\}$. The corresponding odd parity spaces will be denoted $\mathrm{Lg}^{+}$and $\mathrm{Lg}^{-}$, respectively, and the super structure sheaves identified with $\mathscr{F}(L G / G) \otimes \Lambda L \mathfrak{g}^{ \pm}$. In super-string terminology, the former corresponds to the Ramond (R) sector and the latter, the Neveu-Schwarz (NS) one.

We also introduce a super-space extension of the circle $\widetilde{S^{1}}$, with $S^{1}$ as reduced manifold and super structure sheaf $\tilde{\mathscr{F}}\left(\widetilde{S^{1}}\right)$ generated by the angular coordinate $\sigma$ $(\bmod 2 \pi)$ for $S^{1}$ plus an odd nilpotent $\theta$ anticommuting with the odd generators in $\tilde{\mathscr{F}}(\widetilde{L G})$. An element $\tilde{a} \in \tilde{\mathscr{F}}\left(\widetilde{S^{1}}\right)$ may be decomposed into

$$
\tilde{a}(\sigma, \theta)=a(\sigma)+\theta \alpha(\sigma),
$$

where $a(\sigma)$ is an ordinary function on $S^{1}$ while $\alpha(\sigma)$ is odd and either periodic (R) or anti-periodic (NS).

A super loop group element may be identified either by the pair $(g, \psi)$ or, equivalently, the super-loop map

$$
\begin{gathered}
\tilde{g}: \widetilde{S^{1}} \rightarrow G, \\
\tilde{g}:(\sigma, \theta) \mapsto \tilde{g}(\sigma, \theta),
\end{gathered}
$$

denoted formally as

$$
\tilde{g}=(\exp \theta \psi) g=g+\theta \psi g .
$$

The latter consists of an ordinary map $g: S^{1} \rightarrow G$ defining the loop group element 
$g \in L G$, together with a consistent sheaf homomorphism

$$
\begin{aligned}
\tilde{g}^{*}: \mathscr{F}(G) & \rightarrow \tilde{\mathscr{F}}\left(\widetilde{S^{1}}\right), \\
\tilde{g}^{*}: f & \mapsto f \circ g+\theta d f\left(R_{g^{*}} \psi \circ \circ,\right.
\end{aligned}
$$

where $R_{g(\sigma)^{*}} \psi(\sigma)$ denotes right translation of $\left.\psi(\sigma) \in T G\right|_{\text {Id }}$ to $g(\sigma)$. Composition $\tilde{h} \tilde{g}$ of elements $\tilde{h}, \tilde{g} \in \widetilde{L G}$ is defined by the homomorphism obtained by composing the sequence $^{1}$

$$
(\tilde{h} \tilde{g})^{*}: \mathscr{F}(G) \rightarrow \mathscr{F}(G) \otimes \mathscr{F}(G) \stackrel{\tilde{h}^{*} \otimes \tilde{g}^{*}}{\longrightarrow} \tilde{\mathscr{F}}\left(\widetilde{S^{1}}\right) \otimes \tilde{F}\left(\widetilde{S^{1}}\right) \rightarrow \tilde{\mathscr{F}}\left(\widetilde{S^{1}}\right),
$$

where the first map is comultiplication in the sheaf $\mathscr{F}(G)$ associated to group multiplication in $G$, the second is the tensor product of the homomorphisms $h^{*}$ and $g^{*}$, and the third is multiplication of decomposable elements of $\tilde{\mathscr{F}}\left(\widetilde{S^{1}}\right) \otimes \tilde{\mathscr{F}}\left(\widetilde{S}^{1}\right)$ followed by summation.

In the notation (2.1), this just corresponds to developing the product $\tilde{h} \tilde{g}$ formally in the obvious way. Denoting the components of $\tilde{h}$ as $(h, \phi)$, we have

$$
(h+\theta \phi h)(g+\theta \psi g)=h g+\theta(\phi h g+h \psi g),
$$

or, equivalently,

$$
(h, \phi) \cdot(g, \psi)=\left(h g, \phi+h \psi h^{-1}\right) .
$$

Thus, pointwise, $\widetilde{L G}$ appears as the semi-direct product $\widetilde{L G} \times \widetilde{L g}^{ \pm}$, the latter considered an additive abelian subgroup. The identity element is

$$
\widetilde{\mathrm{Id}}=(\mathrm{Id}, 0)
$$

and the inverse is

$$
\tilde{g}^{-1}=g^{-1}-\theta g^{-1} \psi
$$

or, equivalently

$$
(g, \psi)^{-1}=\left(g^{-1},-g^{-1} \psi g\right) .
$$

Left and right translation are denoted

$$
\begin{aligned}
& L_{\tilde{h}}(\tilde{g})=\tilde{h} \tilde{g}, \\
& R_{\tilde{h}}(\tilde{g})=\tilde{g} \tilde{h},
\end{aligned}
$$

or

$$
\begin{aligned}
& L_{(h, \phi)}(g, \psi)=\left(h g, \phi+h \psi h^{-1}\right), \\
& R_{(h, \phi)}(g, \psi)=\left(g h, \psi+g \phi g^{-1}\right),
\end{aligned}
$$

and the adjoint action is

$$
\widetilde{\operatorname{Ad}_{\tilde{h}}}(\tilde{g})=\tilde{h} \tilde{g} \tilde{h}^{-1},
$$

\footnotetext{
${ }^{1}$ This sheaf theoretic formulation of the super loop group composition rule (2.4) was suggested to us by $\mathrm{S}$. Shnider
} 
or, in components

$$
\widetilde{\operatorname{Ad}}_{(h, \phi)}(g, \psi)=\left(h g h^{-1}, \phi+h \psi h^{-1}+\left(h g h^{-1}\right) \phi\left(h g^{-1} h^{-1}\right)\right) .
$$

The corresponding Lie super algebra $\widetilde{L g}$ is identified with the super tangent space at the identity or, equivalently, the sum $L \mathfrak{g}+L \mathfrak{g}^{ \pm}$, with suitably defined Lie bracket. We again introduce pairs $(X, \xi)$ of even and odd generators, $X \in L \mathfrak{g}, \xi \in L \mathfrak{g}^{ \pm}$, the second regarded as a linear element of $\Lambda\left(\mathrm{Lg}^{ \pm}\right)$. A super-algebra element may equivalently be written

$$
\tilde{X}(\sigma, \theta)=X(\sigma)+\theta \xi(\sigma)
$$

with an interpretation in terms of super loops similar to (2.2). The adjoint representation in super space notation is

$$
\widetilde{\operatorname{Ad}}_{\tilde{g}}(\tilde{X})=\tilde{g} \tilde{X} \tilde{g}^{-1}
$$

or, equivalently, in component notation

$$
\widetilde{\operatorname{Ad}}_{(g, \psi)}(X, \xi)=\left(g X g^{-1}, g \xi g^{-1}+\left[\psi, g X g^{-1}\right]\right) .
$$

Taking the super tangent vector to a curve through the identity, the infinitesimal form of (2.10) becomes

$$
\widetilde{\operatorname{ad}}_{\tilde{X}}(\tilde{Y})=[\tilde{X}, \tilde{Y}]=[X, Y]+\theta([X, \eta]-[Y, \xi])
$$

or, equivalently

$$
\widetilde{\operatorname{ad}}_{(X, \xi)}(Y, \eta)=[(X, \xi),(Y, \eta)]=([X, Y],[X, \eta]-[Y, \xi]),
$$

where $\tilde{Y} \equiv Y+\theta \eta$.

The super algebra $\widetilde{L g}$ may also be represented as vector fields on $\widetilde{L G}$, i.e. derivations of the super sheaf $\widetilde{\mathscr{F}}(\widetilde{L G})$. As in the even case, left and right-invariant vector fields are determined either by translation of a super tangent vector at the identity or differentiation of the right and left translation formulas $(2.5 \mathrm{a}, \mathrm{b})$, respectively, along a curve through the identity. The homomorphism

$$
V: L g \rightarrow \tilde{\chi}(L G) \equiv \operatorname{der} \tilde{\mathscr{F}}(L G)
$$

to the Lie algebra of super derivations obtained by differentiating the left translation formula $(2.7 \mathrm{a})$ is defined by

or

$$
V:(X, \xi) \mapsto V_{(X, \xi)}
$$

where

$$
V: \quad \tilde{X} \mapsto V_{\tilde{X}},
$$

$$
V_{\tilde{X}}=-\left[\left\langle X g, \frac{\delta}{\delta g}\right\rangle+\left\langle\xi+[X, \psi], \frac{\delta}{\delta \psi}\right\rangle\right]
$$

is the corresponding right invariant vector field. The notation $\langle X g, \delta / \delta g\rangle$ signifies a functional derivation obtained by pairing the right invariant vector field $X g$ on $L G$ with the functional differential $\delta / \delta g$ at any point $g \in L G$, while $\langle\xi+[X, \psi], \delta / \delta \psi\rangle$ similarly signifies pairing the (odd parity) loop algebra element $\xi+[X, \psi] \in L^{ \pm}$ with the functional differential $\delta / \delta \psi$ on $\mathrm{Lg}^{ \pm}$. The sign in (2.13) is chosen so that 
(2.12) defines a homomorphism rather than an anti-homomorphism:

$$
\left[V_{\tilde{X}}, V_{\tilde{Y}}\right]=V_{[\tilde{X}, \tilde{Y}]} .
$$

Similarly, we define a second homomorphism

$$
W: \widetilde{L g} \rightarrow \tilde{\chi}(\widetilde{L G})
$$

corresponding to the infinitesimal right translation (2.7b) by

$$
\begin{aligned}
& W:(X, \xi) \mapsto W_{(X, \xi)}, \\
& W: \quad \tilde{X} \mapsto W_{\tilde{X}},
\end{aligned}
$$

where

$$
W_{\tilde{X}}=\left\langle g X, \frac{\delta}{\delta g}\right\rangle+\left\langle g \xi g^{-1}, \frac{\delta}{\delta \psi}\right\rangle
$$

is the associated left-invariant vector field, satisfying similarly:

$$
\left[W_{\tilde{X}}, W_{\tilde{Y}}\right]=W_{[\tilde{X}, \tilde{Y}]} .
$$

We assume henceforth that $g$ is endowed with a non-degenerate Ad-invariant metric $b: \mathfrak{g} \times \mathfrak{g} \rightarrow \mathbb{R}$ allowing an identification of $\mathfrak{g}$ with its dual $\mathfrak{g}^{*}$. The superalgebra $L \mathfrak{g}$ may similarly be identified as a dense subspace of the dual $\widetilde{L g}^{*}$, with typical elements denoted by a pair $\left(\mu \in L \mathrm{~g}^{ \pm}, m \in L \mathrm{~g}\right)$ and the embedding $\widetilde{\mathrm{Lg}} \hookrightarrow \widetilde{\mathrm{Lg}^{*}}$ defined by the dual pairing:

$$
\langle(\mu, m),(X, \xi)\rangle \equiv \frac{1}{2 \pi} \int_{0}^{2 \pi} d \sigma[b(m(\sigma), X(\sigma))-b(\mu(\sigma), \xi(\sigma))] .
$$

Identifying the element $(\mu, m) \in \widetilde{L g^{*}}$ with the odd parity dual super-loop element

$$
\tilde{\mu} \equiv \mu+\theta m,
$$

the pairing (2.18) may be expressed succinctly as:

$$
\langle\tilde{\mu}, \tilde{X}\rangle=\frac{1}{2 \pi} \int_{0}^{2 \pi} d \sigma \int d \theta b(\tilde{\mu}, \tilde{X}),
$$

where the odd integral $\int d \theta$ is understood in the usual Berezinian sense, i.e. as picking out the highest (i.e. $\theta$-linear) terms in the integrand. In general, we only consider elements of $\widetilde{\mathrm{Lg}^{*}}$ that may be so identified with elements of $L \mathfrak{g}$ through the parity reversing map

$$
m+\theta \mu \rightarrow \mu+\theta m \in \widetilde{L g^{*}} .
$$

(This reversal of parity is essential for the use of the Berezinian integral in dual pairing to assure invariance under supersymmetry transformations.)

To proceed further, it is useful to introduce, as usual, two odd derivations on the super sheaf $\left.\tilde{\mathscr{F}} \widetilde{S^{1}}\right)$ :

$$
q \equiv \frac{\partial}{\partial \theta}-\theta \frac{\partial}{\partial \sigma},
$$




$$
\mathscr{D} \equiv \frac{\partial}{\partial \theta}+\theta \frac{\partial}{\partial \sigma}
$$

which, together with the even derivation

$$
\partial \equiv \frac{\partial}{\partial \sigma}
$$

generate the left and right super-translation algebras

$$
\begin{aligned}
{[q, q] } & =-2 \partial, \quad[q, \partial]=0 \\
{[\mathscr{D}, \mathscr{D}] } & =2 \partial, \quad[\mathscr{D}, \partial]=0 \\
{[q, \mathscr{D}] } & =0 .
\end{aligned}
$$

By standard conventions, the operator $q$ is viewed as generating the (odd) supersymmetry transformations, while $\mathscr{D}$ is used in constructing supersymmetric quantities containing derivatives. We view $\mathscr{D}$ as defining the odd tangent space distribution that determines the super-conformal structure on $\widetilde{S^{1}}$ (cf. Sect. 4). In superspace terms, the supersymmetry transformations act on $\widetilde{\mathrm{Lg}}$ and $\widetilde{\mathrm{Lg}^{*}}$ by:

$$
\delta_{\varepsilon}(\tilde{X})=\varepsilon q(\tilde{X}), \quad \delta_{\varepsilon}(\tilde{\mu})=\varepsilon q(\tilde{\mu}),
$$

or, in component form:

$$
\begin{array}{rlrl}
\delta_{\varepsilon}(X) & =\varepsilon \xi, & & \delta_{\varepsilon}(\mu)=\varepsilon m, \\
\delta_{\varepsilon}(\xi) & =-\varepsilon X^{\prime}, & \delta_{\varepsilon}(m)=-\varepsilon \mu^{\prime},
\end{array}
$$

where $\varepsilon$ is considered as an odd "parameter." The pairing $(\widetilde{2.18})$ is clearly invariant under such transformations.

Under the identification (2.19), the super coadjoint action, defined by:

$$
\left\langle\widetilde{\operatorname{Ad}}_{\tilde{g}}^{*}(\tilde{\mu}), \tilde{X}\right\rangle \equiv\left\langle\tilde{\mu}, \widetilde{\operatorname{Ad}}_{\tilde{g}^{-1}}(\tilde{X})\right\rangle
$$

coincides formally with the adjoint action in superspace terms

$$
\widetilde{\operatorname{Ad}}_{\tilde{g}}^{*}(\tilde{\mu})=\tilde{g} \tilde{\mu} \tilde{g}^{-1}
$$

but, in components, reverses the rôles of even and odd parts:

$$
\widetilde{\mathrm{Ad}_{(g, \psi)}^{*}}(\mu, m)=\left(g \mu g^{-1}, g m g^{-1}+\left[\psi, g \mu g^{-1}\right]\right) .
$$

(Note: The second term $\left[\psi, g \mu g^{-1}\right]$, though involving a Lie bracket of two odd elements of the algebra does not vanish, but should rather be understood in terms of dual pairing via the ad-invariance of $b$; i.e.

$$
b\left(\left[\psi, g \mu g^{-1}\right], X\right)=b\left(\psi,\left[g \mu g^{-1}, X\right]\right) .
$$

The bracket $[\xi, \eta]$ of two odd elements so interpreted should not be confused with the superalgebra bracket $[(0, \xi),(0, \eta)]$ which does, indeed, vanish.)

Using the above conventions, we define a super-group 1-cocycle $\widetilde{C}: \widetilde{L G} \rightarrow \widetilde{L g}^{*}$ by

$$
\tilde{C}(\tilde{g})=\mathscr{D}(\tilde{g}) \tilde{g}^{-1},
$$


i.e.

$$
\langle\tilde{C}(\tilde{g}), \tilde{X}\rangle \equiv \frac{1}{2 \pi} \int_{0}^{2 \pi} d \sigma \int d \theta b\left(\mathscr{D}(\tilde{g}) \tilde{g}^{-1}, \tilde{X}\right) .
$$

In component terms, this may be expressed

$$
\widetilde{C}(g, \psi)=\left(\psi, g^{\prime} g^{-1}+\frac{1}{2}[\psi, \psi]\right)
$$

(where $g^{\prime} \equiv d g / d \sigma$ ) or, equivalently

$$
\langle\tilde{C}(g, \psi),(X, \xi)\rangle=\frac{1}{2 \pi} \int_{0}^{2 \pi} d \sigma\left[b\left(g^{\prime} g^{-1}+\frac{1}{2}[\psi, \psi], X\right)-b(\psi, \xi)\right] .
$$

The cocycle relation

$$
\tilde{C}(\tilde{h}, \tilde{g})=\widetilde{\mathrm{Ad}_{\tilde{h}}^{*}} \tilde{C}(\tilde{g})+C(\tilde{h})
$$

is easily verified.

The corresponding super-algebra (scalar-valued) 2-cocycle

$$
\tilde{c}: \widetilde{L g} \times \widetilde{L g} \rightarrow \mathbb{R}
$$

is defined by

$$
\tilde{c}(\tilde{X}, \tilde{Y})=\frac{1}{2 \pi} \int_{0}^{2 \pi} d \sigma \int d \theta b(\tilde{X}, \mathscr{D} \tilde{Y}),
$$

or equivalently, in component form:

$$
\tilde{c}((X, \xi),(Y, \eta))=\frac{1}{2 \pi} \int_{0}^{2 \pi} d \sigma\left[b\left(X, Y^{\prime}\right)+b(\xi, \eta)\right] .
$$

The invariance of $\tilde{c}$ under the super-symmetry transformations (2.24) follows from the superspace form $(2.30)$ and the commutation relation $(2.23 \mathrm{c})$. The centrally extended super algebra $\widetilde{\mathrm{Lg}}^{\wedge}$ is identified with the space

$$
\widetilde{L g}+\mathbb{R} \equiv\{(\tilde{X}, a)\}
$$

with super Lie bracket

$$
[(\tilde{X}, a),(\tilde{Y}, b)]=([\tilde{X}, \tilde{Y}], \tilde{c}(\tilde{X}, \tilde{Y}))
$$

or, in component notation

$$
[(X, \xi, a),(Y, \eta, b)]=([X, Y],[X, \eta]-[Y, \xi], \tilde{c}((X, \xi),(Y, \eta))) .
$$

The map $(\widetilde{2.19})$ and pairing (2.18) may be extended to identify $\widetilde{\mathrm{Lg}^{\wedge}}$ as a dense subspace of $\widetilde{\mathrm{Lg}^{\wedge}}$, with elements of the form $(\tilde{\mu}, a)$ and dual pairing:

$$
\langle(\tilde{\mu}, a),(\tilde{X}, b)\rangle^{\wedge} \equiv\langle\tilde{\mu}, \tilde{X}\rangle+a b .
$$

The extended adjoint action is defined by:

$$
\widetilde{\operatorname{Ad}}_{\tilde{g}}(\tilde{X}, a)=\left(\operatorname{Ad}_{\tilde{g}}(\tilde{X}), a+\left\langle\tilde{C}\left(\tilde{g}^{-1}\right), \tilde{X}\right\rangle\right)
$$


or, in component form:

$$
\widetilde{\operatorname{Ad}}_{(g, \psi)}(X, \xi, a)=\left(g X g^{-1}, g \xi g^{-1}+\left[\psi, g X g^{-1}\right], a+\left\langle\widetilde{C}\left(g^{-1},-\psi\right),(X, \xi)\right\rangle\right) .
$$

(As usual, it is not necessary to define a central extension $\widetilde{L G}{ }^{\wedge}$ of the group $\widetilde{L G}$ to define its adjoint action, since this only depends on the image in $\widetilde{L G}$. It is possible, however, just as in the even case [PS], to define $\widetilde{L G}^{\wedge}$ as a circle bundle over $\widetilde{L G}$.) The corresponding extended coadjoint action is

$$
\widetilde{\operatorname{Ad}}_{\tilde{g}}^{\wedge} *(\tilde{\mu}, a)=\left(\widetilde{\operatorname{Ad}}_{\tilde{g}}^{*}(\tilde{\mu})+a \mathscr{D}(\tilde{g}) \tilde{g}^{-1}, a\right)
$$

or, in components

$\widetilde{\operatorname{Ad}}_{(g, \psi)}^{\wedge}(\mu, m, a)=\left(g \mu g^{-1}+a \psi, g m g^{-1}+a g^{\prime} g^{-1}+\left[\psi, g \mu g^{-1}\right]+\frac{1}{2} a[\psi, \psi], a\right)$.

\section{The Phase Space $\widetilde{L G} / G$ and the $\widetilde{L \mathfrak{g}^{\wedge}}$ Moment Map}

Proceeding analogously to the even case ([H], [PS]), a functional differential 2-form on $\overparen{L G}$ is defined by

$$
\tilde{\omega} \equiv-\frac{k}{4 \pi} \int_{0}^{2 \pi} d \sigma \int d \theta b\left(\delta\left(\mathscr{D}(\tilde{g}) \tilde{g}^{-1}\right) \hat{\prime} \delta \tilde{g} \tilde{g}^{-1}\right),
$$

where $k$ is an arbitrary real constant, $\delta$ signifies functional exterior derivative and $b(\hat{,})$ means simultaneous exterior product of functional differential forms and scalar product on $\mathfrak{g}$. In component form, this may be written

$$
\tilde{\omega}=-\frac{k}{4 \pi} \int_{0}^{2 \pi} d \sigma b\left(\delta\left(g^{\prime} g^{-1}\right) \wedge \delta g g^{-1}\right)+\frac{k}{4 \pi} \int_{0}^{2 \pi} d \sigma b(\delta \psi \wedge \hat{,} \delta \psi) .
$$

The meaning of this 2 -form and its relation to the even case is clarified by the following:

Lemma 3.1. Evaluating $\tilde{\omega}$ on a pair of right-invariant vector fields gives

$$
\tilde{\omega}\left(V_{\tilde{X}}, V_{\tilde{Y}}\right)=k \tilde{c}(\tilde{X}, \tilde{Y})+k\langle\tilde{C}(\tilde{g}),[\tilde{X}, \tilde{Y}]\rangle,
$$

while on a pair of left-invariant ones

$$
\tilde{\omega}\left(W_{\tilde{X}}, W_{\tilde{Y}}\right)=k \tilde{c}(\tilde{X}, \tilde{Y}) .
$$

Proof. Expressing $V_{\tilde{X}}$ and $V_{\tilde{Y}}$ as a sum of parts tangential to the even $(B)$ and odd $(F)$ parts of $L G$, we have:

$$
V_{\tilde{X}}=V_{\tilde{X}}^{B}+V_{\tilde{X}}^{F}, \quad V_{\tilde{Y}}=V_{\tilde{Y}}^{B}+V_{\tilde{Y}}^{F},
$$

where:

$$
\begin{array}{ll}
V_{\tilde{X}}^{B}=-\left\langle X \psi, \frac{\delta}{\delta g}\right\rangle, & V_{\tilde{X}}^{F}=-\left\langle\xi+[X, \psi], \frac{\delta}{\delta \psi}\right\rangle, \\
V_{\tilde{Y}}^{\boldsymbol{B}}=-\left\langle Y \psi, \frac{\delta}{\delta g}\right\rangle, & V_{\tilde{Y}}^{F}=-\left\langle\eta+[Y, \psi], \frac{\delta}{\delta \psi}\right\rangle,
\end{array}
$$


and

$$
\tilde{X} \equiv X+\theta \xi, \quad \tilde{Y} \equiv Y+\theta \eta
$$

Evaluating $\tilde{\omega}$ on the parts, we find:

$$
\begin{aligned}
& \tilde{\omega}\left(V_{\tilde{X}}^{\boldsymbol{B}}, V_{\tilde{Y}}^{\boldsymbol{B}}\right)=\frac{k}{2 \pi} \int_{0}^{2 \pi} d \sigma\left[b\left(X, Y^{\prime}\right)+b\left(g^{\prime} g^{-1},[X, Y]\right)\right], \\
& \tilde{\omega}\left(V_{\tilde{X}}^{F}, V_{\tilde{Y}}^{F}\right)=\frac{k}{2 \pi} \int_{0}^{2 \pi} d \sigma\left[b(\xi, \eta)+b([X, \eta]-[Y, \xi], \psi)+\frac{1}{2} b([X, Y],[\psi, \psi])\right], \\
& \tilde{\omega}\left(V_{\tilde{X}}^{F}, V_{\tilde{Y}}^{B}\right)=\tilde{\omega}\left(V_{\tilde{X}}^{B}, V_{\tilde{Y}}^{F}\right)=0,
\end{aligned}
$$

where the ad-invariance of $b$, the graded Jacobi identity and integration by parts have been used. Summing thus gives

$$
\begin{aligned}
\tilde{\omega}\left(V_{\tilde{X}}, V_{\tilde{Y}}\right)= & \frac{k}{2 \pi} \int_{0}^{2 \pi} d \sigma\left[b\left(X, Y^{\prime}\right)+b(\xi, \eta)\right] \\
& +\frac{k}{2 \pi} \int_{0}^{2 \pi} d \sigma\left[b\left(g^{\prime} g^{-1}+\frac{1}{2}[\psi, \psi],[X, Y]\right)+b([X, \eta]-[Y, \xi], \psi)\right]
\end{aligned}
$$

which is just $\overparen{3.2}$ ) in component form.

Similarly, splitting

$$
W_{\tilde{X}}=W_{\tilde{X}}^{B}+W_{\tilde{X}}^{F}, \quad W_{\tilde{Y}}=W_{\tilde{Y}}^{B}+W_{\tilde{Y}}^{F},
$$

where

$$
\begin{aligned}
& W_{\tilde{X}}^{\boldsymbol{B}}=\left\langle g X, \frac{\delta}{\delta g}\right\rangle, \quad W_{\tilde{X}}^{F}=\left\langle g \xi g^{-1}, \frac{\delta}{\delta \psi}\right\rangle, \\
& W_{\tilde{Y}}^{\boldsymbol{B}}=\left\langle g Y, \frac{\delta}{\delta g}\right\rangle, \quad W_{\tilde{Y}}^{F}=\left\langle g \eta g^{-1}, \frac{\delta}{\delta \psi}\right\rangle,
\end{aligned}
$$

and evaluating on each part gives

$$
\begin{aligned}
\tilde{\omega}\left(W_{\tilde{X}}^{B},\left(W_{\tilde{Y}}^{B}\right)\right. & =\frac{k}{2 \pi} \int_{0}^{2 \pi} d \sigma b\left(X, Y^{\prime}\right), \\
\tilde{\omega}\left(W_{\tilde{X}}^{F}, W_{\tilde{Y}}^{F}\right) & =\frac{k}{2 \pi} \int_{0}^{2 \pi} d \sigma b(\xi, \eta), \\
\tilde{\omega}\left(W_{\tilde{X}}^{F}, W_{\tilde{Y}}^{B}\right) & =\tilde{\omega}\left(W_{\tilde{X}}^{B}, W_{\tilde{Y}}^{F}\right)=0,
\end{aligned}
$$

Thus, summing gives

$$
\tilde{\omega}\left(W_{\tilde{X}}, W_{\tilde{Y}}\right)=\frac{k}{2 \pi} \int_{0}^{2 \pi} d \sigma\left[b\left(X, Y^{\prime}\right)+b(\xi, \eta)\right],
$$

which is (3.3) in component form.

The meaning of $\tilde{\omega}$ is thus very simple; decomposing $T \widetilde{L G}$ into even and odd tangent spaces, the restriction of $\tilde{\omega}$ to the even part is just $k$ times the even part 
of the cocycle when evaluated on left invariant vector fields on $L G$, while on the odd part it is just the scalar product determined by $k b$.

Now identify $G \subset \widetilde{L G}$ as the subgroup of even constant loops $\{(g \in G, 0)\}$. As in the even case ([PS], $[\mathrm{H}]$ ), we have the following theorem, showing that $\tilde{\omega}$ may be projected to define a sympletic $\widetilde{L G}$-invariant form on the coset space $\widetilde{L G} / G$.

Theorem 3.2. The form $\tilde{\omega}$ is left $\widetilde{L G}$-invariant and right $G$-invariant. It is closed, $\delta \tilde{\omega}=0$, and its kernel distribution is the vertical distribution corresponding to the fibration $\pi: \widetilde{L G} \rightarrow \widetilde{L G} / G$.

Proof. Left $\widetilde{L G}$-invariance is verified since

$$
\begin{aligned}
L_{\tilde{h}}^{*} \tilde{\omega} & =-\frac{k}{4 \pi} \int_{0}^{2 \pi} d \sigma \int d \theta b\left(\delta\left(\mathscr{D}(\tilde{h}) \tilde{h}^{-1}\right)+\tilde{h} \delta\left(\mathscr{D}(\tilde{g}) \tilde{g}^{-1}\right) \tilde{h}^{-1} \hat{,} \tilde{h}(\delta \tilde{g}) \tilde{g}^{-1} \tilde{h}^{-1}\right) \\
& =\tilde{\omega}
\end{aligned}
$$

the first equality following from the Leibniz rule for the derivation $\mathscr{D}$, the second from the Ad-invariance of $b$ and the fact that $\delta\left(\mathscr{D}(\tilde{h}) \tilde{h}^{-1}\right.$ ) vanishes (since $\tilde{h}$ is constant on $L G)$. Right $G$-invariance follows by inspection. To verify that $\tilde{\omega}$ is closed, evaluate $\delta \tilde{\omega}$ on a triplet of left-invariant vector fields, using (3.3) and (2.17):

$$
\delta \tilde{\omega}\left(W_{\tilde{X}}, W_{\tilde{Y}}, W_{\tilde{Z}}\right)=k\left(W_{\tilde{X}} \tilde{c}(\tilde{Y}, \tilde{Z})-\tilde{c}([\tilde{X}, \tilde{Y}], \tilde{Z})\right)+\text { cyclic permutations. }
$$

The first term vanishes because $\tilde{c}(\tilde{Y}, \tilde{Z})$ is a constant on $\widetilde{L G}$ and the second summed over cyclic permutations vanishes by the cocycle identity. To compute the kernel distribution, evaluate $\tilde{\omega}$ on a pair $\left(W_{\tilde{X}}, W_{\tilde{Y}}\right)$. If

$$
\tilde{\omega}\left(W_{\tilde{X}}, W_{\tilde{Y}}\right)=\frac{k}{2 \pi} \int_{0}^{2 \pi} d \sigma \int d \theta b(\tilde{X}, \mathscr{D}(\tilde{Y}))=0
$$

for all $\tilde{Y} \in \widetilde{L g}$, it follows that $\mathscr{D}(\tilde{X})$ vanishes (weakly) and hence $\tilde{X}$ is of the form $(X, 0)$, where $X \in \mathfrak{g}$ is a constant loop. But such $W_{\tilde{X}}$ span the vertical tangent distribution of the fibration $\pi: \widetilde{L G} \rightarrow \widetilde{L G} / G$.

In the following, $\tilde{\omega}$ will also denote the super-symplectic form on $\widetilde{L G} / G$ obtained by projection. The left $\widetilde{L G}$-action defined by $(\widetilde{2.7 \mathrm{a}})$ projects to a left action, also denoted by $L_{\tilde{h}}$, on the quotient $\widetilde{L G} / G$ :

$$
L_{\tilde{h}}(\tilde{g} G)=\tilde{h} \tilde{g} G .
$$

The corresponding homomorphism

$$
\begin{aligned}
& V: \widetilde{L g} \rightarrow \tilde{\chi}(\widetilde{L G} / G) \equiv \operatorname{der} \tilde{\mathscr{F}}(\widetilde{L G} / G), \\
& V: \tilde{X} \mapsto V_{\tilde{X}}
\end{aligned}
$$

to the algebra of derivations of the sub-sheaf $\tilde{\mathscr{F}}(\widetilde{L G} / G) \subset \tilde{\mathscr{F}}(\widetilde{L G})$ of right $G$-invariant elements, obtained by differentiating (3.4) along a curve through $\tilde{h}=\widetilde{\mathrm{Id}}$, is obtained by restricting $V_{\tilde{X}}$ as defined by $(2.13)$ to $\tilde{\mathscr{F}}(\widetilde{L G / G})$. Evaluating the inner product with $\tilde{\omega}$ gives: 


$$
\begin{aligned}
\left.V_{\tilde{X}}\right\rfloor \tilde{\omega} & =-\frac{k}{2 \pi} \int_{0}^{2 \pi} d \sigma\left[b\left(\delta\left(g^{\prime} g^{-1}\right), X\right)+b(\xi+[X, \psi], \delta \psi)\right] \\
& =-\delta\left(J_{\tilde{X}}^{L}\right)
\end{aligned}
$$

where

$$
\tilde{J}_{\tilde{X}}^{L}=\frac{k}{2 \pi} \int_{0}^{2 \pi} d \sigma\left[b\left(g^{\prime} g^{-1}+\frac{1}{2}[\psi, \psi], X\right)-b(\psi, \xi)\right] .
$$

Thus, $V_{\tilde{X}}$ is a Hamiltonian vector field generated by $-\tilde{J}_{\tilde{X}}^{L}$. Defining the map

$$
\tilde{J}^{L}: \widetilde{L G} / G \rightarrow \widetilde{L g^{*}}
$$

by

$$
\tilde{J}^{L}: \tilde{g} G \mapsto k \mathscr{D}(\tilde{g}) \tilde{g}^{-1}=k \tilde{C}(\tilde{g})
$$

or, in components,

$$
\tilde{J}^{L}:(g G, \psi) \mapsto k\left(\psi, g^{\prime} g^{-1}+\frac{1}{2}[\psi, \psi]\right),
$$

we have

$$
\tilde{J}_{\tilde{X}}^{L}=\left\langle\tilde{J}^{L}, \tilde{X}\right\rangle=\frac{k}{2 \pi} \int_{0}^{2 \pi} d \sigma \int d \theta b\left(\mathscr{D}(\tilde{g}) \tilde{g}^{-1}, \tilde{X}\right) .
$$

Thus, $\widetilde{J}^{L}$ is just $k$ times the cocycle $(2.27)$ projected to $\widetilde{L G} / G$, where it becomes a 1-1 moment map generating the left $L G$-action (3.4) by Hamiltonian flows. Evaluating the Poisson brackets gives

$$
\left\{\tilde{J}_{\tilde{X}}^{L}, \tilde{J}_{\tilde{Y}}^{L}\right\}=\tilde{J}_{[\tilde{X}, \tilde{Y}]}^{L}+k \tilde{c}(\tilde{X}, \tilde{Y}),
$$

and therefore $\tilde{J}^{L}$ is non-equivariant, with cocycle $k \tilde{c}$. In finite form, the nonequivariance is given by $k \tilde{C}$ :

$$
\tilde{J}^{L} \circ L_{\tilde{h}}=\operatorname{Ad}_{\tilde{h}}^{*} \circ \tilde{J}^{L}+k \tilde{C}(\tilde{h})
$$

which is equivalent to the cocycle relation (2.29).

Summarizing, we have the following result:

Theorem 3.3. The left $\widetilde{L G}$-action on $\widetilde{L G} / G$ is Hamiltonian, and generated by the

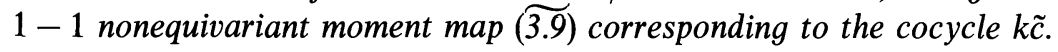

Following the usual procedure, the moment map $\tilde{J}^{L}$ may be extended to an equivariant one

$$
\begin{aligned}
& \tilde{J}^{L \wedge}: \widetilde{L G} / G \rightarrow \widetilde{L g^{\wedge}}{ }^{*}, \\
& \tilde{J}^{L \wedge}: \quad \tilde{g} G \mapsto(k \widetilde{C}(\tilde{g}), k)
\end{aligned}
$$

to the dual of the centrally extended algebra $\widetilde{L g}^{\wedge}$ determined by the cocycle $\tilde{c}$. Defining

$$
\tilde{J}_{(\tilde{X}, a)}^{L \wedge} \equiv\left\langle\tilde{J}^{L \wedge},(\tilde{X}, a)\right\rangle^{\wedge}=\tilde{J}_{\tilde{X}}^{L}+k a,
$$

we get

$$
\left\{\tilde{J}_{(\tilde{X}, a)}^{L \wedge}, \tilde{J}_{(\tilde{Y}, b)}^{L \wedge}\right\}=\tilde{J}_{[(\tilde{\tilde{X}}, a),(\tilde{Y}, b)]}^{L},
$$


and

$$
\widetilde{J}^{L \wedge} \circ L_{\tilde{h}}=\widetilde{\operatorname{Ad}}_{\hat{h}}^{\wedge} \circ \widetilde{J}^{L \wedge} .
$$

Thus $\tilde{J}^{L \wedge}$ is an equivariant moment map providing a super Poisson bracket realization of the centrally extended algebra $\widetilde{L g}$.

Define the (Kostant-Kirillov) super symplectic form on coadjoint orbits of $L \mathfrak{g}^{\wedge}$ by:

$$
\left.\tilde{\omega}_{\text {orb }}\left(\widetilde{\operatorname{ad}}_{\hat{\bar{X}}} * \widetilde{\operatorname{ad}}_{\hat{\bar{Y}}}^{*}\right)\right|_{(\tilde{\mu}, a)}=\langle\tilde{\mu},[\tilde{X}, \tilde{Y}]\rangle+a \tilde{c}(\tilde{X}, \tilde{Y}),
$$

where $\widetilde{\operatorname{ad}}_{\hat{\bar{X}}} *$ and $\widetilde{\operatorname{ad}}_{\hat{Y}} *$ are interpreted as vector fields on the coadjoint orbit through $(\tilde{\mu}, a) \in \widetilde{L g}^{\wedge *}$ induced by the infinitesimal coadjoint action. It follows from the equivariance of $\tilde{J}^{L \wedge}$ that the pull-back of $\tilde{\omega}_{\text {orb }}$ restricted to the coadjoint orbit $\mathcal{O}_{(0, k)} \subset \widetilde{L g}^{\wedge} *$ through the point $(0, k)$ satisfies

$$
\left.\tilde{J}^{L \wedge *} \tilde{\omega}_{\text {orb }}\right|_{\mathcal{O}_{(0, k)}}\left(V_{\tilde{X}}, V_{\tilde{Y}}\right)=k\langle\tilde{C}(\tilde{g}),[\tilde{X}, \tilde{Y}]\rangle+k \tilde{c}(\tilde{X}, \tilde{Y})
$$

when evaluated on a pair of vector fields induced by the infinitesimal left action. By Lemma (3.1), Eq. (3.2), this coincides with the evaluation of $\tilde{\omega}$ and hence, we have

$$
\left.\tilde{J}^{L \wedge *} \tilde{\omega}_{\text {orb }}\right|_{\Theta_{(0, k)}}=\tilde{\omega} .
$$

Summarizing these results, we have the following theorem.

Theorem 3.4. The extended moment map $\widetilde{J}^{\llcorner\wedge}: \widetilde{L G} / G \rightarrow \widetilde{L g}^{\wedge *}$ defined in (3.12) is an equivariant symplectomorphism between $\widetilde{L G} / G$ and the extended coadjoint orbit $\mathcal{O}_{(0, k)} \subset \widetilde{L g}^{\wedge *}$ with respect to the orbital super symplectic form $\tilde{\omega}_{\text {orb }}$.

We emphasize that all the results of this section, when expressed in superspace terms, appear as the natural extensions of the corresponding results for ordinary loop groups and algebras. There is an underlying richer structure, however, that becomes apparent in component form due to the presence of the anti-commuting terms $\{\psi\}$. It is worthwhile noting that, under the identification of the super structure sheaf $\tilde{\mathscr{F}}(\widetilde{L G} / G)$ with $\mathscr{F}(L G / G) \otimes \Lambda\left(L g^{ \pm}\right)$, the super symplectic structure restricts, according to (3.1), on the sub-sheaf $\chi_{B} \subset \operatorname{der} \tilde{\mathscr{F}}(\widetilde{L G} / G)$ of derivations that annihilate $\mathbf{I} \otimes \Lambda\left(L \mathfrak{g}^{ \pm}\right)$, to the standard symplectic form ([H], [PS])

$$
\omega_{B} \equiv-\frac{k}{4 \pi} \int_{0}^{2 \pi} d \sigma b\left(\delta\left(g^{\prime} g^{-1}\right) \wedge(\delta g) g^{-1}\right)
$$

for $L G / G$. On the subsheaf $\chi_{F} \subset \operatorname{der} \tilde{\mathscr{F}}(\widetilde{L G} / G)$ of derivations that annihilate $\mathscr{F}(L G / G) \otimes \mathbf{I}$, it restricts to

$$
\omega_{F} \equiv \frac{k}{4 \pi} \int_{0}^{2 \pi} d \sigma b(\delta \psi \wedge \delta \psi)
$$

Thus, the Poisson bracket induced on $\Lambda\left(L \mathfrak{g}^{ \pm}\right)$is just the standard graded commutator in terms of the Clifford algebra multiplication on $\Lambda\left(L \mathfrak{g}^{ \pm}\right)$associated to the metric

$$
\frac{1}{2 \pi} \int_{0}^{2 \pi} d \sigma b(,)
$$


Elements of the subsheaves $\mathscr{F}(L G / G) \otimes \mathbf{I}$ and $\mathbf{I} \otimes \Lambda\left(L \mathfrak{g}^{ \pm}\right)$mutually Poisson commute. The moment map (3.9) may also be split into a sum:

$$
\widetilde{J}^{L}=\widetilde{J}_{B}^{L}+\widetilde{J}_{F}^{L}
$$

of two moment maps:

$$
\begin{aligned}
& \tilde{J}_{B}^{L}: \widetilde{L G} / G \rightarrow \widetilde{L g^{*}}, \\
& \tilde{J}_{B}^{L}:(g, \psi) \mapsto\left(0, k g^{\prime} g^{-1}\right),
\end{aligned}
$$

and

$$
\begin{aligned}
& \widetilde{J}_{F}^{L}: \widetilde{L G} / G \rightarrow \widetilde{L g^{*},} \\
& \widetilde{J}_{F}^{L}:(g, \psi) \mapsto k\left(\psi, \frac{1}{2}[\psi, \psi]\right)
\end{aligned}
$$

that mutually commute. The first part $\tilde{J}_{B}^{L}$ has vanishing odd component, while its even part is the usual (even) moment map on $L G / G$ :

$$
\begin{aligned}
& \tilde{J}_{B}^{L}: L G / G \rightarrow L g^{*} \\
& \tilde{J}_{B}^{L}: \quad g B \mapsto k g^{\prime} g^{-1} .
\end{aligned}
$$

The second part $\tilde{J}_{F}^{L}$ projects to a Fermionic moment map on $L g^{ \pm}$whose odd part is just the identity map (the "chiral Fermi field" $\psi$ ) while the even part (the Fermi "current"):

$$
\begin{aligned}
& J_{F}^{L}: L \mathfrak{g}^{ \pm} \rightarrow L \mathfrak{g}^{*}, \\
& J_{F}^{L}: \quad \psi \mapsto \frac{1}{2} k[\psi, \psi]
\end{aligned}
$$

satisfies

$$
\left\{J_{F, X}^{L}, J_{F, Y}^{L}\right\}=J_{F,[X, Y]}^{L},
$$

where

$$
\begin{aligned}
J_{F, X}^{L} \equiv\left\langle J_{F}^{L}, X\right\rangle \\
=\frac{k}{4 \pi} \int_{0}^{2 \pi} d \sigma b([\psi, \psi], X(\sigma)), \\
X \in L \mathrm{~g} .
\end{aligned}
$$

Thus, $J_{F}^{L}$ is an equivariant moment map generating the natural (conjugation) action of $L G$ on $L \mathrm{~g}^{ \pm}$. The two parts $V_{\tilde{X}}^{B}$ and $V_{\tilde{X}}^{F}$ appearing in the proof of Lemma 3.1 are just the Hamiltonian vector fields on $L G / G$ and $L g^{ \pm}$, respectively, induced by the two moment maps $\widetilde{J}_{B}^{L}$ and $\widetilde{J}_{F}^{L}$. The fermionic moment map $\widetilde{J}_{F}^{L}$ projects to $L \mathfrak{g}^{ \pm}$, but is non-equivariant:

$$
\left\{\tilde{J}_{F, \tilde{X}}^{L}, \tilde{J}_{F, \tilde{Y}}^{L}\right\}=\tilde{J}_{F,[\tilde{X}, \tilde{Y}]}^{L}+k c_{F}(\tilde{X}, \tilde{Y}),
$$

where

$$
\tilde{J}_{F, \tilde{X}}^{L} \equiv\left\langle\tilde{J}_{F}^{L}, \tilde{X}\right\rangle, \quad \tilde{J}_{F, \tilde{Y}}^{L} \equiv\left\langle\tilde{J}_{F}^{L}, \tilde{Y}\right\rangle
$$

and

$$
c_{F}(\tilde{X}, \tilde{Y}) \equiv \frac{1}{2 \pi} \int_{0}^{2 \pi} d \sigma b(\xi, \eta)
$$


for

$$
\tilde{X}=X+\theta \xi, \quad \tilde{Y}=Y+\theta \eta
$$

This again gives rise to an extended moment map

$$
\begin{aligned}
& \tilde{J}_{\boldsymbol{F}}^{L \wedge}: L \mathfrak{g}^{ \pm} \rightarrow{\widetilde{L g^{\wedge}}}^{*} \equiv \widetilde{L g^{*}}+\mathbb{R}, \\
& \tilde{J}_{\boldsymbol{F}}^{L \wedge}: \quad \psi \mapsto k\left(\psi, \frac{1}{2}[\psi, \psi], 1\right),
\end{aligned}
$$

embedding $L \mathfrak{g}^{ \pm}$as a coadjoint orbit in the dual of a central extension $\widetilde{L g} \hat{F} \sim \widetilde{L g} \oplus \mathbb{R}$ of $\widetilde{\mathrm{Lg}}$. However, $\widetilde{\mathrm{gg}_{\boldsymbol{F}}}$ is not the superalgebra $\widetilde{\mathrm{Lg}^{\wedge}}$, but rather the central extension of $\widetilde{L g}$ defined on the same space $\widetilde{L g} \oplus \mathbb{R}$ by the nonsupersymmetric Lie bracket:

$$
[(\tilde{X}, a),(\tilde{Y}, b)]=\left([\tilde{X}, \tilde{Y}], c_{F}(\tilde{X}, \tilde{Y})\right),
$$

i.e. using the fermionic part of the cocycle alone.

\section{Hamiltonian Action of $\widetilde{\operatorname{diff}^{c}} S^{1}$ on $\widetilde{L G} / G$ and $\widetilde{L g^{\wedge}}{ }^{*}$}

Denote by diff $S^{1}$ the algebra of superderivations of $\tilde{\mathscr{F}}\left(\tilde{S}^{1}\right)$. A typical element may be expressed relative to the $(\partial, \mathscr{D})$ basis as

$$
\hat{a}=\hat{a} \partial+\widetilde{\beta} \mathscr{D},
$$

where

$$
\begin{aligned}
& \tilde{a}=a(\sigma)+\theta \alpha(\sigma), \\
& \tilde{\beta}=\beta(\sigma)+\theta b(\sigma) .
\end{aligned}
$$

As in the super loop algebra, the even terms $a(\sigma), b(\sigma)$ are periodic in $\sigma$, while the odd terms $\alpha(\sigma), \beta(\sigma)$ may be periodic or anti-periodic. The superconformal structure on $\widetilde{S^{1}}$ is determined by the $(0 \mid 1)$-dimensional non-integrable distribution spanned by $\mathscr{D}$. The subalgebra $\widetilde{\text { diff }^{c}} S^{1}$ of superconformal derivations is defined to be those preserving $\mathscr{D}$, i.e.

$$
\widetilde{\operatorname{diff}^{c}} S^{1} \equiv\left\{\hat{a} \in \widetilde{\operatorname{diff}} S^{1},[\hat{a}, \mathscr{D}] \propto \mathscr{D}\right\}
$$

This means that a superderivation $\hat{a}$ is in $\widetilde{\operatorname{diff}^{c}} S^{1}$ if it is of the form:

$$
\hat{a} \equiv \tilde{a} \partial+\frac{1}{2} \mathscr{D}(\tilde{a}) \mathscr{D},
$$

i.e.

$$
\begin{aligned}
& \tilde{\beta}=\frac{1}{2} \mathscr{D} \tilde{a}, \\
& \beta=\frac{1}{2} \alpha, \quad b=\frac{1}{2} a^{\prime} .
\end{aligned}
$$

The commutator of two such elements $\hat{\tilde{a}}_{1}, \hat{\tilde{a}}_{2} \in \widetilde{\operatorname{diff}^{c}} S^{1}$ is

$$
\begin{aligned}
{\left[\hat{a}_{1}, \hat{a}_{2}\right] } & =\widehat{\tilde{a}_{1} \tilde{a}_{2}^{\prime}-\tilde{a}_{2} \tilde{a}_{1}^{\prime}+\frac{1}{2} \mathscr{D}\left(\tilde{a}_{1}\right) \mathscr{D}\left(\tilde{a}_{2}\right)} \\
& \equiv \widehat{\left[\tilde{a}_{1}, \tilde{a}_{2}\right]} .
\end{aligned}
$$

There is a natural homomorphism

$$
U: \widetilde{\operatorname{diff}} S^{1} \rightarrow \operatorname{der} \tilde{\mathscr{F}}(\widetilde{L G} / G)
$$


to the algebra of right $G$-invariant derivations of $\tilde{\mathscr{F}}(\widetilde{L G})$ defined by:

$$
\begin{gathered}
U: \hat{a} \mapsto U_{\tilde{a}}, \\
U_{\tilde{a}} \equiv-\left[\left\langle\delta_{\tilde{a}}(g), \frac{\delta}{\delta g}\right\rangle+\left\langle\delta_{\tilde{a}}(\psi), \frac{\delta}{\delta \psi}\right\rangle\right],
\end{gathered}
$$

where $\delta_{\tilde{a}}(g)$ and $\delta_{\tilde{a}}(\psi)$ are defined by

$$
\hat{a} \tilde{g} \equiv \delta_{\tilde{a}}(g)+\theta \delta_{\tilde{a}}(\psi g) .
$$

This corresponds to the infinitesimal version of super-loop reparametrizations. Explicitly, we have

$$
\begin{aligned}
\delta_{\tilde{a}}(g) & =a g^{\prime}+\beta \psi g \\
\delta_{\tilde{a}}(\psi) & =(\alpha-\beta) g^{\prime} g^{-1}+\frac{1}{2} \beta[\psi, \psi]+a \psi^{\prime}+b \psi
\end{aligned}
$$

For $\hat{a} \equiv \hat{\tilde{a}} \in \widetilde{\operatorname{diff}}^{c} S^{1}$, this reduces to

$$
\begin{aligned}
& \delta_{\tilde{a}}(g)=a g^{\prime}+\frac{1}{2} \alpha \psi g, \\
& \delta_{\tilde{a}}(\psi)=\frac{1}{2} \alpha\left(g^{\prime} g^{-1}+\frac{1}{2}[\psi, \psi]\right)+a \psi^{\prime}+\frac{1}{2} a^{\prime} \psi .
\end{aligned}
$$

Since $L \mathfrak{g}$ may be identified with $\mathfrak{g} \otimes \widetilde{\mathscr{F}} \widetilde{\left(S^{1}\right)}$, derivations $\hat{a} \in \widetilde{\operatorname{diff}} S^{1}$ may be extended linearly to $\widetilde{\mathrm{Lg}}$, thereby defining the semi-direct sum $\widetilde{\operatorname{diff}} S^{1} \ltimes \widetilde{\mathrm{Lg}}$. The homomorphisms defined by Eqs. (2.12), (2.13), (2.15), (2.16) and (4.8) then define representations of $\overparen{\text { diff }} S^{1} \ltimes \widetilde{L g}$ in terms of functional vector fields on $\widetilde{L G} / G$.

Proposition 4.1. The functional vector fields $U_{\tilde{a}}$, $V_{\tilde{X}}$ and $W_{\tilde{X}}$ defined by the homomorphisms (2.13), (2.16) and (4.8) satisfy

$$
\begin{aligned}
{\left[U_{\tilde{a}}, V_{\tilde{X}}\right] } & =V_{\tilde{a}(\tilde{X})}, \\
{\left[U_{\tilde{a}}, W_{\tilde{X}}\right] } & =W_{\tilde{a}(\tilde{X})},
\end{aligned}
$$

and therefore the maps

$$
\begin{aligned}
U & \times V: \widetilde{\operatorname{diff}} S^{1} \ltimes \widetilde{L g} \rightarrow \operatorname{der} \tilde{\mathscr{F}}(\widetilde{L G}), \\
U & \times V: \quad(\hat{a}, \tilde{X}) \mapsto U_{\tilde{a}}+V_{\tilde{X}}, \\
U & \times W: \widetilde{\operatorname{diff}} S^{1} \ltimes \widetilde{L g} \rightarrow \operatorname{der} \tilde{\mathscr{F}}(\widetilde{L G}), \\
U & \times W: \quad(\hat{a}, \tilde{X}) \mapsto U_{\tilde{a}}+W_{\tilde{X}}
\end{aligned}
$$

are Lie algebra homomorphisms.

Proof. Direct computation.

The next theorem gives two further equivalent characterizations of the subalgebra $\widetilde{\operatorname{diff}}^{c} S^{1}$.

Theorem 4.2. The following conditions are all equivalent.

i) $\hat{a} \in \widetilde{\operatorname{diff}} S^{1}$ is in $\widetilde{\operatorname{diff}}^{c} S^{1}$.

ii) The element $\hat{a} \in \operatorname{diff} S^{1}$ preserves the cocycle $\tilde{c}$ :

$$
\tilde{c}(\hat{a}(\tilde{X}), \tilde{Y})+\tilde{c}(\tilde{X}, \hat{a}(Y))=0 .
$$


iii) The vector field $U_{\hat{a}} \in \operatorname{der} \tilde{\mathscr{F}}(\widetilde{L G} / G)$ preserves the symplectic form $\tilde{\omega}$,

$$
\mathscr{L}_{U_{a}}(\tilde{\omega})=0 .
$$

[Note: The equivalence (i)↔(ii) is given, in a slightly different formulation, in $[\mathrm{KT}]$.]

Proof. From the definition $(\widetilde{2.30})$ of $\tilde{c}$ we obtain, integrating by parts and using the commutation relations (2.23),

$$
\tilde{c}(\hat{a}(\tilde{X}), \tilde{Y})+\tilde{c}(\tilde{X}, \hat{a}(Y))=\frac{1}{2 \pi} \int_{0}^{2 \pi} d \sigma \int d \theta(2 \tilde{\beta}-\mathscr{D}(a)) b(\mathscr{D}(\tilde{X}), \mathscr{D}(\tilde{Y})) .
$$

Therefore, (4.16) is satisfied for all $X, \tilde{Y}$ if and only if (4.5) is; i.e. $\hat{a}=\hat{\hat{a}} \in \widetilde{\operatorname{diff}}^{c} S^{1}$. Evaluating $\mathscr{L}_{U_{\hat{a}}}(\tilde{\omega})$ on a pair $\left(W_{\tilde{X}}, W_{\tilde{Y}}\right)$ of left-invariant vector fields on $\widetilde{L G}$, we find

$$
\begin{aligned}
\mathscr{L}_{U_{a}}(\tilde{\omega})\left(W_{\tilde{X}}, W_{\tilde{Y}}\right) & =U_{\hat{a}}\left(\tilde{\omega}\left(W_{\tilde{X}}, W_{\tilde{Y}}\right)\right)-\tilde{\omega}\left(\left[U_{\hat{a}}, W_{\tilde{X}}\right], W_{\tilde{Y}}\right)-\tilde{\omega}\left(W_{\tilde{X}},\left[U_{\hat{a}}, W_{\tilde{Y}}\right]\right) \\
& =-k \tilde{c}(\hat{a} \tilde{X}, \tilde{Y})-k \tilde{c}(\tilde{X}, \hat{a} \tilde{Y}),
\end{aligned}
$$

where (3.3) and (4.13) have been used. Thus, Eqs. (4.16) and (4.17) are equivalent.

Henceforth, only the subalgebra $\widetilde{\operatorname{diff}^{c}} S^{1}$ of superconformal vector fields on $\widetilde{S}^{1}$ will be considered, and the restriction of the homomorphisms (4.14), (4.15) to the semi-direct sum $\widetilde{\text { diff }}^{c} S^{1} \ltimes \widetilde{\mathrm{Lg}}$. Elements $\hat{a} \in \widetilde{\mathrm{diff}}^{c} S^{1}$ will normally be designated by the superfunction $\tilde{a} \in \tilde{\mathscr{F}}\left(\widetilde{S^{1}}\right)$ entering in the coordinate representation (4.5). The dual diff ${ }^{c} S^{1 *}$ will be identified with the space of (odd-parity) differential forms on $\widetilde{S^{1}}$ annihilating the (0|1)-dimensional distribution spanned by $\mathscr{D}$ :

$$
\widetilde{\operatorname{diff}^{c}} S^{1 *} \equiv\{\hat{\tilde{\lambda}} \equiv \tilde{\lambda}(d \sigma-\theta d \sigma)\},
$$

where

$$
\begin{aligned}
& \tilde{\lambda}=\lambda(\sigma)+\theta l(\sigma), \\
& l(\sigma+2 \pi)=l(\sigma), \\
& \lambda(\sigma+2 \pi)= \pm \lambda(\sigma) .
\end{aligned}
$$

Elements of $\widetilde{\operatorname{diff}^{c}} S^{1 *}$ will be designated by the superfunction $\tilde{\lambda}$ entering in the coordinate representation (4.18). The dual pairing

$$
\widetilde{\operatorname{diff}^{c}} S^{1 *} \times \widetilde{\operatorname{diff}^{c}} S^{1} \rightarrow \mathbb{R}
$$

is again defined by Berezinian integration

$$
\langle\tilde{\lambda}, \tilde{a}\rangle_{v} \equiv \frac{1}{2 \pi} \int_{0}^{2 \pi} d \sigma \int d \theta \hat{\lambda}(\hat{a})=\frac{1}{2 \pi} \int_{0}^{2 \pi} d \sigma \int d \theta \tilde{\lambda} \tilde{a},
$$

or, expressing $\tilde{\lambda}$ and $\tilde{a}$ in terms of their components $(\lambda, l)$ and $(a, \alpha)$, respectively,

$$
\langle(\lambda, l),(a, \alpha)\rangle_{v}=\frac{1}{2 \pi} \int_{0}^{2 \pi} d \sigma[l a-\lambda \alpha] .
$$


A super cocycle $\tilde{c}_{v}$ may be defined by

$$
\begin{aligned}
\tilde{c}_{v}\left(\tilde{a}_{1}, \tilde{a}_{2}\right) & =\frac{1}{2 \pi} \int_{0}^{2 \pi} d \sigma \int d \theta \tilde{a}_{1}^{\prime} \mathscr{D}\left(\tilde{a}_{2}^{\prime}\right) \\
& =\frac{1}{2 \pi} \int_{0}^{2 \pi} d \sigma\left[a_{1}^{\prime} a_{2}^{\prime \prime}+\alpha_{1}^{\prime} \alpha_{2}^{\prime}\right],
\end{aligned}
$$

where

$$
\tilde{a}_{1}=a_{1}+\theta \alpha_{1}, \quad \tilde{\alpha}_{2}^{\prime}=a_{2}+\theta \alpha_{2} .
$$

This then defines the central extension

$$
\widetilde{\operatorname{diff}^{c}} S^{1 \wedge}=\widetilde{\operatorname{diff}^{c}} S^{1}+\mathbb{R}=\{(\tilde{a}, r)\}
$$

with super Lie bracket

$$
\left[\left(\tilde{a}_{1}, r_{1}\right),\left(\tilde{a}_{2}, r_{2}\right)\right]=\left(\left[\tilde{a}_{1}, \tilde{a}_{2}\right], \tilde{c}_{v}\left(\tilde{a}_{1}, \tilde{a}_{2}\right)\right) .
$$

The next theorem is the main result of this section, showing that the vector fields $U_{\hat{a}}$ defining the representation of $\widetilde{\operatorname{diff}}^{c} S^{1}$ on $\tilde{\mathscr{F}}(\widetilde{L G} / G)$ are derived from an equivariant moment map.

Theorem 4.3. The symplectic vector fields $U_{a}$, $\hat{a} \in \operatorname{diff}^{c} S^{1}$ are Hamiltonian, with

$$
\left.U_{a}\right\rfloor \tilde{\omega}=-\delta \tilde{J}_{a},
$$

where

$$
\begin{aligned}
\tilde{J}_{\tilde{a}} & \equiv\langle\tilde{J}, \tilde{a}\rangle_{v}=\frac{k}{4 \pi} \int_{0}^{2 \pi} d \sigma \int d \theta \tilde{a}\left[b(\tilde{\mathscr{E}}, \mathscr{D} \tilde{\mathscr{E}})-\frac{1}{3} b(\tilde{\mathscr{E}},[\tilde{\mathscr{E}}, \tilde{\mathscr{E}}])\right], \\
\tilde{\mathscr{E}} & \equiv \mathscr{D}(\tilde{g}) \tilde{g}^{-1}=\frac{1}{k} \tilde{J}^{L} .
\end{aligned}
$$

The moment map

$$
\begin{aligned}
& \tilde{J}: \widetilde{L G} / G \rightarrow{\widetilde{\operatorname{diff}^{c}}}^{1 *}, \\
& \tilde{J}: \quad \tilde{g} G \mapsto \frac{k}{2}\left[b(\widetilde{\mathscr{E}}, \mathscr{D} \widetilde{\mathscr{E}})-\frac{1}{3} b(\widetilde{\mathscr{E}},[\widetilde{\mathscr{E}}, \widetilde{\mathscr{E}}])\right]
\end{aligned}
$$

is equivariant:

$$
\left\{\tilde{J}_{\tilde{a}}, \tilde{J}_{\tilde{b}}\right\}=\tilde{J}_{[\tilde{a}, \tilde{b}]} .
$$

In component form:

$$
\tilde{J}_{\tilde{a}}=\frac{k}{4 \pi} \int_{0}^{2 \pi} d \sigma\left\{a\left[b(E, E)+b\left(\psi^{\prime}, \psi\right)-b(E,[\psi, \psi])\right]+\alpha\left[b(E, \psi)-\frac{1}{3} b(\psi,[\psi, \psi])\right]\right\},
$$

where

$$
\tilde{\mathscr{E}}=\psi+\theta E
$$




$$
E=g^{\prime} g^{-1}+\frac{1}{2}[\psi, \psi]
$$

The Poisson brackets with the generators $\tilde{J}_{\tilde{X}}^{L}$ of left translations satisfy:

$$
\left\{\tilde{J}_{\tilde{a}}, \tilde{J}_{\tilde{X}}^{L}\right\}=\tilde{J}_{\tilde{a}(\tilde{X})}^{L},
$$

and hence the pair $\left\{\tilde{J}, \tilde{J}^{L \wedge}\right\}$ define a Poisson bracket realization of $\operatorname{diff} S^{1} \ltimes L \mathfrak{g}^{\wedge}$ on $\widetilde{L G} / G$.

Proof. A direct computation from the definition (4.8), (4.11a,b) of $U_{a}$ and the formula (4.26) for $\widetilde{J}_{\tilde{a}}$.

In fact, this result may be derived another way that lends further insight into the structure of the moment map (4.28). Define a map

by

$$
\widetilde{J}^{\varphi}: \widetilde{L g}^{\wedge} * \widetilde{\operatorname{diff}^{c}} S^{1 *}
$$

$$
\tilde{J}^{\mathscr{S}}(\tilde{\mu}, r) \equiv \frac{1}{2 r}\left[b(\tilde{\mu}, \mathscr{D} \tilde{\mu})-\frac{1}{3 r} b(\tilde{\mu},[\tilde{\mu}, \tilde{\mu}])\right] .
$$

Then, from (4.27) and (4.28), we see that $\widetilde{J}$ is just the composition of $\widetilde{J}^{L \wedge}$, with $\widetilde{J}^{\mathscr{S}}$,

$$
\tilde{J}=\tilde{J}^{\mathscr{S}} \circ \tilde{J}^{L \wedge}
$$

i.e. we have a factorization described by the diagram:

$$
\begin{aligned}
& \widetilde{L G} / G \stackrel{\tilde{J}}{\longrightarrow} \widetilde{\operatorname{diff}}^{c} S^{1 *} \\
& \widetilde{J}^{L \wedge} \widetilde{L \mathfrak{g}}^{\wedge *}
\end{aligned}
$$

The point is that $\tilde{J}^{\mathscr{S}}$ is also a Poisson map with respect to the Lie-Poisson brackets defined on $\widetilde{\mathrm{Lg}^{\wedge *} \text { by }}$

$$
\begin{gathered}
\left.\{f, g\}\right|_{(\tilde{\mu}, r)}=\left\langle\tilde{\mu},\left[\frac{\delta f}{\delta \tilde{\mu}}, \frac{\delta g}{\delta \tilde{\mu}}\right]\right\rangle+r \tilde{c}\left(\frac{\delta f}{\delta \tilde{\mu}}, \frac{\delta g}{\delta \tilde{\mu}}\right) \\
f, g \in \tilde{\mathscr{F}}\left(\widetilde{L g^{\wedge}}\right), \quad(\tilde{\mu}, r) \in \widetilde{L g}^{\wedge *},
\end{gathered}
$$

and on $\widetilde{\operatorname{diff}^{c}} S^{1 *}$ by

$$
\begin{gathered}
\left.\{F, G\}\right|_{\tilde{\lambda}}=\left\langle\tilde{\lambda},\left[\frac{\delta F}{\delta \tilde{\lambda}}, \frac{\delta G}{\delta \tilde{\lambda}}\right]\right\rangle_{v}, \\
F, G \in \tilde{\mathscr{F}}\left(\widetilde{\operatorname{diff}^{c}} S^{1 *}\right), \quad \tilde{\lambda} \in \widetilde{\operatorname{diff}}^{c} S^{1 *} .
\end{gathered}
$$

This is stated in the following theorem, from which Theorem (4.3) may be deduced as a corollary.

Theorem 4.4. The map $\widetilde{J}^{\mathscr{y}}: \widetilde{\mathrm{Lg}^{\wedge}} \rightarrow{\widetilde{\mathrm{diff}^{c}}}^{c} S^{1 *}$ preserves the Poisson brackets (4.34) and (4.35), i.e.

$$
\begin{gathered}
\left\{F \circ \widetilde{J}^{\mathscr{S}}, G \circ \widetilde{J}^{\mathscr{S}}\right\}=\{F, G\} \circ \widetilde{J}^{\mathscr{S}}, \\
F, G \in \mathscr{F}\left(\widetilde{\operatorname{diff}} S^{1 *}\right) .
\end{gathered}
$$


In particular, on linear elements

$$
\begin{aligned}
F & =\langle\tilde{\lambda}, \tilde{a}\rangle_{v}, \quad G=\langle\tilde{\lambda}, \tilde{b}\rangle, \\
F \circ \tilde{J}^{\mathscr{S}} & =\left\langle\tilde{J}^{\mathscr{S}}, \tilde{a}\right\rangle_{v} \equiv \tilde{J}_{\tilde{a}}^{\mathscr{S}} \\
G \circ \tilde{J}^{\mathscr{S}} & =\left\langle\tilde{J}^{\mathscr{S}}, \tilde{b}\right\rangle_{v} \equiv \tilde{J}_{\bar{b}}^{\mathscr{S}},
\end{aligned}
$$

we have

$$
\left\{\tilde{J}_{\tilde{a}}^{\mathscr{S}}, \tilde{J}_{\tilde{b}}^{\mathscr{L}}\right\}=\tilde{J}_{[\tilde{a}, \tilde{b}]}^{\mathscr{S}},
$$

i.e. the dual map

$$
\begin{aligned}
& \tilde{J}^{\mathscr{S}^{*}}: \widetilde{\operatorname{diff}} S^{1} \rightarrow \widetilde{\mathscr{F}}\left(\widetilde{\mathrm{Lg}^{\wedge}}{ }^{*}\right), \\
& \tilde{J}^{\mathscr{S}^{*}}: \quad \tilde{a} \mapsto \widetilde{J}_{\tilde{a}}^{\mathscr{\mathcal { S }}}
\end{aligned}
$$

is a homomorphism to the Poisson algebra on $\tilde{\mathscr{F}}\left(\widetilde{L g}^{\wedge} *\right)$.

Proof. It is sufficient to prove the result in its linear form (4.39), since the derivation property of Poisson brackets then implies it is valid for all $F, G \in \tilde{\mathscr{F}}\left(\operatorname{diff} S^{1 *}\right.$ ). Computing the functional derivative of $\widetilde{J}_{\tilde{a}}^{\mathscr{L}}$ gives

$$
\frac{\delta \widetilde{J}_{\tilde{a}}^{\mathscr{\varphi}}}{\delta \tilde{\mu}}=\frac{1}{2 r}\left[2 \tilde{a} \mathscr{D}(\tilde{\mu})+\mathscr{D}(\tilde{a}) \tilde{\mu}-\frac{1}{r} \tilde{a}[\tilde{\mu}, \tilde{\mu}]\right],
$$

which should be viewed as an element of $\mathrm{Lg}$. Substituting in (4.34) and using the graded Jacobi identity gives

$$
\begin{aligned}
\left\{\tilde{J}_{\tilde{a}}^{\mathscr{S}}, \tilde{J}_{\tilde{b}}^{\mathscr{S}}\right\}= & \frac{1}{4 r^{2}}\langle\tilde{\mu},[2 \tilde{a} \mathscr{D}(\tilde{\mu})+\mathscr{D}(\tilde{a}) \tilde{\mu}, 2 \tilde{b} \mathscr{D} \tilde{\mu}+\mathscr{D}(\tilde{b}) \tilde{\mu}\rangle \\
& +\frac{1}{4 r}\langle\mathscr{D}(2 \tilde{b} \mathscr{D}(\tilde{\mu})+\mathscr{D}(\tilde{b}) \tilde{\mu}), 2 \tilde{a} \mathscr{D}(\tilde{\mu})+\mathscr{D}(\tilde{a}) \tilde{\mu}\rangle \\
& -\frac{1}{4 r^{2}}\{\langle\mathscr{D}(b[\tilde{\mu}, \tilde{\mu}]), 2 \tilde{a} \mathscr{D}(\tilde{\mu})+\mathscr{D}(\tilde{a}) \tilde{\mu}\rangle \\
& +\langle\mathscr{D}(2 \tilde{b} \mathscr{D}(\tilde{\mu})+\mathscr{D}(\tilde{b}) \tilde{\mu}), \tilde{a}[\tilde{\mu}, \tilde{\mu}]\rangle\} .
\end{aligned}
$$

(Note that since $\tilde{\mu}$ is odd, the Jacobi identity implies that $[\tilde{\mu},[\tilde{\mu}, \tilde{\mu}]]$ vanishes and hence, by Ad-invariance, so do all terms of the form $\langle\tilde{\mu},[[\tilde{\mu}, \tilde{\mu}], X]\rangle$.) Integrating by parts and using identities of the type

$$
\begin{aligned}
b\left(\tilde{\mu}, \tilde{\mu}^{\prime}\right) & =b(\mathscr{D}(\tilde{\mu}), \mathscr{D}(\tilde{\mu}))-\mathscr{D}(b(\tilde{\mu}, \mathscr{D}(\tilde{\mu}))), \\
b\left(\mathscr{D}(\tilde{\mu}), \tilde{\mu}^{\prime}\right) & =\frac{1}{2} \mathscr{D}(b(\mathscr{D}(\tilde{\mu}), \mathscr{D}(\tilde{\mu}))),
\end{aligned}
$$

we find the factor with coefficient $1 / 4 r$ reduces to:

$$
\left\langle\left(2 \tilde{a} \tilde{b}^{\prime}-2 \tilde{a}^{\prime} \tilde{b}+\mathscr{D}(\tilde{a}) \mathscr{D}(\tilde{b})\right) \tilde{\mu}, \mathscr{D}(\tilde{\mu})\right\rangle,
$$

while the one with coefficient $1 / 4 r^{2}$ becomes:

$$
-\frac{2}{3}\left\langle\tilde{\mu},\left(\tilde{a} \tilde{b}^{\prime}-\tilde{b} \tilde{a}^{\prime}+\frac{1}{2} \mathscr{D}(\tilde{a}) \mathscr{D}(\tilde{b})\right)[\tilde{\mu}, \tilde{\mu}]\right\rangle .
$$


Adding gives the result

$$
\begin{aligned}
\left\{\tilde{J}_{\tilde{a}}^{\mathscr{S}}, \tilde{J}_{\tilde{b}}^{\mathscr{b}}\right\} & =\frac{1}{2 r}\left\langle\tilde{\mu},\left(\tilde{a} \tilde{b}^{\prime}-\tilde{b} \tilde{a}^{\prime}+\frac{1}{2} \mathscr{D}(\tilde{a}) \mathscr{D}(\tilde{b})\right)\left(\mathscr{D}(\tilde{\mu})-\frac{1}{3 r}[\tilde{\mu}, \tilde{\mu}]\right)\right\rangle \\
& =\tilde{J}_{[\tilde{a}, \tilde{b}]}^{\mathscr{C}} \cdot \square
\end{aligned}
$$

We see from Theorem 4.4 that $\tilde{J}^{\mathscr{S}}$ is also a moment map, generating representations of diff $S^{1}$ as Hamiltonian vector fields on the symplectic leaves of $\widetilde{L g}^{\wedge} *$; i.e. on the coadjoint orbits. In view of Theorem 3.4 , which identifies $\overparen{L G} / G$ with such coadjoint orbits via the moment map $\widetilde{J}^{L \wedge}$, we see that Theorem 4.3 follows as a corollary to Theorem 4.4 .

To obtain further insight into the moment map (4.28), note that, if we decompose the $\widetilde{\mathrm{Lg}^{*}}$ moment map $\widetilde{J}^{L}$ into Fermi and Bose parts, as in (3.21):

$$
\tilde{J}^{L}=k \psi+\theta\left(J_{B}^{L}+J_{F}^{L}\right)
$$

substitution into (4.28) gives:

$$
\widetilde{J}=\Gamma+\theta L
$$

where

$$
\begin{aligned}
\Gamma & \equiv \frac{1}{2} b\left(J_{B}^{L}+\frac{1}{3} J_{F}^{L}, \psi\right), \\
L & \equiv \frac{1}{2 k}\left[b\left(J_{B}^{L}, J_{B}^{L}\right)+k^{2} b\left(\psi^{\prime}, \psi\right)\right],
\end{aligned}
$$

and $J_{F}^{L}, J_{B}^{L}$ are the Fermi and Bose "currents". (Note that $b\left(J_{F}^{L}, J_{F}^{L}\right)$ vanishes because of the graded Jacobi identity.) Equations (4.43), (4.44) may be viewed as the supersymmetric version of the classical Sugawara formula, which thus differs from the abelian case only by the presence of the additional $\frac{1}{6} b\left(J_{F}^{L}, \psi\right)$ term.

\section{Fourier Analysis}

In this section, we re-express the results of Sects. 3 and 4 in terms of Fourier components. Let $\left\{T_{a}\right\}$ be a basis for $g$, orthonormal with respect to the metric $b$, and satisfying

$$
\left[T_{a}, T_{b}\right]=f_{a b}^{c} T_{c} .
$$

Through the usual identifications $L \mathfrak{g} \subset L \mathfrak{g}^{*}, \mathfrak{g} \sim \mathfrak{g}^{*}, \widetilde{L g} \subset \widetilde{\mathrm{Lg}^{*}}$, we express the odd part of $\tilde{J}^{L}$ (i.e. the Fermi field $\psi$ ) and the bosonic and fermionic contributions to the even part (i.e. the currents $J_{B}^{L}$ and $J_{F}^{L}$ ) as Fourier series:

$$
\begin{aligned}
k \psi & \equiv \sum_{p} \sum_{a} \psi_{a \mid p} T_{a} e^{-i p \sigma}, \\
J_{B}^{L} & \equiv \sum_{n \in \mathbb{Z}} \sum_{a} E_{a \mid n}^{B} T_{a} e^{-i n \sigma}, \\
J_{F}^{L} & \equiv \sum_{n \in \mathbb{Z}} \sum_{a} E_{a \mid n}^{F} T_{a} e^{-i n \sigma},
\end{aligned}
$$

where $p \in \mathbb{Z}$ for periodic $(R)$ boundary conditions and $p \in \mathbb{Z}+\frac{1}{2}$ for anti-periodic 
(NS) ones. The generators of the super loop algebra $\widetilde{L g}$ are then $\left\{\psi_{a \mid p}, E_{a \mid n}\right\}$, where

$$
E_{a \mid n} \equiv E_{a \mid n}^{B}+E_{a \mid n}^{F}
$$

are the Fourier components of the full current

$$
J^{L} \equiv J_{B}^{L}+J_{F}^{L}=\sum_{n \in \mathbf{Z}} \sum_{a} E_{a \mid n} T_{a} e^{-i n \sigma} .
$$

From Eq. (3.25), it follows that the Fourier components of the Fermi current are expressible as

$$
E_{a \mid n}^{F}=\frac{1}{2 k} \sum_{p} \sum_{b c} f_{a b}^{c} \psi_{b \mid p} \psi_{c \mid n-p}
$$

in terms of the Fermi field components.

The non-zero Poisson brackets following from Eqs. (3.10), (3.26) and (3.28) are then

$$
\begin{aligned}
& \left\{\psi_{a \mid p}, \psi_{b \mid q}\right\}=k \delta_{a b} \delta_{p+q, 0}, \\
& \left\{E_{a \mid n}, \psi_{b \mid q}\right\}=\sum_{c} f_{a b}^{c} \psi_{c \mid n+q}, \\
& \left\{E_{a \mid n}, E_{b \mid m}\right\}=\sum_{c} f_{a b}^{c} E_{c \mid n+m}-i n k \delta_{n+m, 0} \delta_{a, b}, \\
& \left\{E_{a \mid n}^{B}, E_{b \mid m}^{B}\right\}=\sum_{c} f_{a b}^{c} E_{c \mid n+m}^{B}-i n k \delta_{n+m, 0} \delta_{a, b}, \\
& \left\{E_{a \mid n}^{F}, \psi_{b \mid q}\right\}=\sum_{c} f_{a b}^{c} \psi_{c \mid n+q}, \\
& \left\{E_{a \mid n}^{F}, E_{b \mid m}^{F}\right\}=\sum_{c} f_{a b}^{c} E_{c \mid n+m}^{F} .
\end{aligned}
$$

The boson current components $E_{a \mid n}^{B}$ Poisson commute with both the Fermi field $\psi_{a \mid p}$ and Fermi current components $E_{a \mid n}^{F}$. Thus, the set $\left\{\psi_{a \mid p}, E_{a \mid n}^{F}\right\}$ generates a Poisson bracket realization of $\widetilde{L \mathfrak{g}_{\hat{F}}}$ on the Fermi phase space $L \mathfrak{g}^{ \pm}$, while the set $\left\{E_{a \mid n}^{B}\right\}$ generates a realization of $L \mathfrak{g}^{\wedge}$ (central extension of the ordinary loop algebra with the even part of the cocycle $\tilde{c}$ ) on the Bose phase space $L G / G$. The full set $\left\{\psi_{a \mid p}, E_{a \mid n}\right\}$ generates a Poisson bracket realization of $L \mathfrak{g}^{\wedge}$ on the super phase space $L G / G$. According to the results of Sect. 3, these Fermi, Bose and super phase spaces are all interpretable as coadjoint orbits, within the spaces $\widetilde{L \mathfrak{g}}_{\mathfrak{F}} *, L \mathfrak{g}^{\wedge *}$ and $\widetilde{L g}^{\wedge *}$, respectively.

Next, we turn to the generators of the $\widetilde{\operatorname{diff}}^{c} S^{1}$-action on $\widetilde{L G} / G$. Decomposing the moment map $\tilde{J}$ into even and odd parts as in Eq. (4.42), each of these may be expressed as a Fourier series:

$$
\begin{aligned}
\Gamma & =\sum_{p} \Gamma_{p} e^{-i p \sigma}, \\
L & =\sum_{n \in \mathbf{Z}} L_{n} e^{-i n \sigma},
\end{aligned}
$$

where, again, $p \in \mathbb{Z}$ for periodic (R) boundary conditions and $p \in \mathbb{Z}+\frac{1}{2}$ for antiperiodic (NS) ones. It follows from Eqs. (4.43) and (4.44) that the Fourier 
components for $\Gamma$ and $L$ are expressible in terms of those for $\psi, J_{B}^{L}$ and $J_{F}^{L}$ as:

$$
\begin{aligned}
& \Gamma_{p}=\frac{1}{2 k} \sum_{n} \sum_{a}\left[E_{a \mid n}^{B} \psi_{a \mid p-n}+\frac{1}{3} E_{a \mid n}^{F} \psi_{a \mid p-n}\right], \\
& L_{n}=\frac{1}{2 k}\left\{\sum_{m} \sum_{a}\left[E_{a \mid m}^{B} E_{a \mid n-m}^{B}\right]-\sum_{p} \sum_{a} i p\left[\psi_{a \mid p} \psi_{a \mid n-p}\right]\right\} .
\end{aligned}
$$

This may be interpreted as the classical super Sugawara formula. A comparison with the quantum (i.e. representation theoretic) one of ref. [KT] will be given in Sect. 8. Note that, by combining Eqs. (5.9a, b) with (5.4) and (5.6), we may express the generators $\left\{\Gamma_{p}, L_{n}\right\}$ entirely in terms of the generators $\left\{\psi_{a \mid p}, E_{a \mid n}\right\}$ of the super loop algebra:

$$
\begin{aligned}
\Gamma_{p}= & \frac{1}{2 k} \sum_{n} \sum_{a}\left[E_{a \mid n} \psi_{a \mid p-n}-\frac{1}{3 k} \sum_{q} f_{b c}^{a} \psi_{b \mid q} \psi_{c \mid n-q} \psi_{a \mid p-n}\right], \\
L_{n}= & \frac{1}{2 k} \sum_{m} \sum_{a} E_{a \mid m} E_{a \mid n-m}-\frac{1}{2 k} \sum_{p} \sum_{a} i p \psi_{a \mid p} \psi_{a \mid n-p} \\
& -\frac{1}{2 k^{2}} \sum_{m} \sum_{q} \sum_{a b c} f_{a b}^{c} E_{c \mid m} \psi_{a \mid q-m} \psi_{b \mid n-q} .
\end{aligned}
$$

From Eq. (4.39), we obtain the Poisson bracket relations defining the complexified algebra $\widetilde{\operatorname{diff}}_{\mathbb{C}} S^{1}$ :

$$
\begin{aligned}
& \left\{L_{n}, L_{m}\right\}=-i(n-m) L_{n+m}, \\
& \left\{L_{n}, \Gamma_{p}\right\}=-i\left(\frac{n}{2}-p\right) \Gamma_{n+p}, \\
& \left\{\Gamma_{p}, \Gamma_{q}\right]=\frac{1}{2} L_{p+q} .
\end{aligned}
$$

Finally, from Eq. (4.31), we obtain the Poisson brackets

$$
\begin{aligned}
& \left\{L_{n}, E_{a \mid m}\right\}=m E_{a \mid n+m}, \\
& \left\{L_{n}, \psi_{a \mid p}\right\}=i\left(p+\frac{n}{2}\right) \psi_{a \mid n+p}, \\
& \left\{\Gamma_{p}, E_{a \mid n}\right\}=i \frac{n}{2} \psi_{a \mid n+p}, \\
& \left\{\Gamma_{p}, \psi_{a \mid q}\right\}=\frac{1}{2} E_{a \mid p+q} .
\end{aligned}
$$

Relations $(5.7 \mathrm{a}-\mathrm{c}),(5.11 \mathrm{a}-\mathrm{c}),(5.12 \mathrm{a}-\mathrm{d})$ thus give a Poisson bracket realization of the full super algebra $\widetilde{\operatorname{diff}}_{\mathbb{C}} S^{1} \ltimes \widetilde{L g}^{\wedge}$.

We note that, at the classical level, the $\operatorname{diff}_{\mathbb{C}} S^{1}$ subalgebra $(5.11 \mathrm{a}-\mathrm{c})$ has no central extension. In the following section we shall see, however, that a classical center may be obtained by introducing a slight modification to the representation (4.8). 


\section{Twisted diff $\widetilde{C}^{c} S^{1}$ Action}

Let $\rho: U(1) \rightarrow G$ be a group homomorphism and $\dot{\rho}: \mathbb{R} \rightarrow \mathfrak{g}$ its derivative at the identity. The corresponding element of $\mathbb{R}^{*} \otimes \mathfrak{g}=\mathfrak{g}$ will also be denoted $\dot{\rho}$. The homomorphism defined by restricting (4.8) to $\operatorname{diff}^{c} S^{1}$ may be modified to define a new one

$$
\begin{aligned}
U^{\rho}: \operatorname{diff}^{c} S^{1} & \rightarrow \operatorname{der}_{\mathbb{C}} \tilde{\mathscr{F}}(\widetilde{L G} / G), \\
U^{\rho}: \quad \hat{\tilde{a}} & \mapsto U_{\tilde{a}}^{\rho}, \\
U_{\hat{\tilde{a}}}^{\rho} & \equiv U_{\hat{\tilde{a}}}+V_{\tilde{X}_{\tilde{a}}},
\end{aligned}
$$

where $V_{\tilde{X}_{\tilde{a}}}$ is the projection to $\widetilde{L G} / G$ of the complex-valued right invariant vector field on $\widetilde{L G}$ given by Eq. (2.13), corresponding to the element $\tilde{X}_{\tilde{a}} \in(\widetilde{L g})_{\mathbb{C}}$ defined by:

$$
\tilde{X}_{\tilde{a}} \equiv-i \tilde{a}^{\prime} \dot{\rho} .
$$

This is just the infinitesimal form of the "twisted" super conformal action on $\widetilde{L G} / G$ obtained by combining a super reparametrization with the left translation obtained by composing the Jacobian (exponentiated) with the homomorphism $\rho$. It is straightforward to verify that (6.2) provides a representation of $\widetilde{\text { iiff }^{c}} S^{1}$ in terms of super vector fields on $\widetilde{L G} / G$ :

$$
\left[U_{\tilde{a}}^{\rho}, U_{\tilde{b}}^{\rho}\right]=U_{[\tilde{a}, \tilde{b}]}^{\rho} .
$$

It also follows, since both $U_{\tilde{a}}$ and $V_{\tilde{X}_{\tilde{a}}}$ are Hamiltonian, that $U_{\tilde{a}}^{\rho}$ is as well.

Theorem 6.1. The $\widetilde{\operatorname{diff}^{c}} S^{1}$ representation defined by (6.1) is (infinitesimally) Hamiltonian, with moment map

$$
\widetilde{J}^{\rho}: \widetilde{L G} / G \rightarrow \widetilde{\operatorname{diff}_{\mathbb{C}}^{c}} S^{1 *}
$$

defined by

$$
\widetilde{J}^{\rho}=\widetilde{J}+i b\left(\dot{\rho}, \widetilde{J}^{L^{\prime}}\right),
$$

i.e.

$$
\left.U_{\tilde{a}}^{\rho}\right\rfloor \tilde{\omega}=-\delta\left(J_{\tilde{a}}^{\rho}\right),
$$

where

$$
\tilde{J}_{\tilde{a}}^{\rho} \equiv\left\langle\tilde{J}^{\rho}, \tilde{a}\right\rangle_{v}=\tilde{J}_{\tilde{a}}^{\rho}+\tilde{J}_{\tilde{X}_{\tilde{a}}}^{L}
$$

satisfies the Poisson bracket relations

$$
\left\{\tilde{J}_{\tilde{a}}^{\rho}, \tilde{J}_{\tilde{b}}^{\rho}\right\}=\tilde{J}_{[\tilde{a}, \tilde{b}]}^{\rho}-k \dot{\rho}^{2} \tilde{c}_{v}(\tilde{a}, \tilde{b})
$$

with

$$
\dot{\rho}^{2} \equiv b(\dot{\rho}, \dot{\rho}) .
$$

Proof. Immediate from Theorems 3.3 and 4.4.

Note that the Poisson bracket relations (6.7) imply that the "twisted" moment map $\widetilde{J}^{\rho}$ is non-equivariant, giving a Poisson bracket realization of the centrally 
extended algebra $\widetilde{\operatorname{diff}^{c}} S^{1 \wedge}$ defined in (4.24). Denote by

$$
\widetilde{\operatorname{Vir}} \equiv \widetilde{\operatorname{diff}_{\mathbb{C}}^{c}} S^{1 \wedge} \equiv\left\{\left(\tilde{a} \in \widetilde{\operatorname{diff}_{\mathbb{C}}^{c}} S^{1}, S \in \mathbb{C}\right)\right\}
$$

the complexification of $\operatorname{diff}^{c} S^{1 \wedge}$ (i.e., the Ramond or Neveu-Schwarz algebra, collectively called the super Virasoro algebra). The dual space is:

$$
\begin{aligned}
\widetilde{\text { Vir }^{*}} & =\widetilde{\operatorname{diff}_{\mathbb{C}}^{c}} S^{1 *}+\mathbb{C} \\
& \equiv\left\{(\tilde{\lambda}, r), \tilde{\lambda} \in\left(\operatorname{diff}^{c} S^{1}\right)_{\mathbb{C}}^{*}, r \in \mathbb{C}\right\}
\end{aligned}
$$

with pairing:

$$
\langle(\tilde{\lambda}, r),(\tilde{a}, s)\rangle_{s} \equiv\langle\tilde{\lambda}, \hat{a}\rangle_{v}+r s .
$$

The super Lie-Poisson brackets on $\widetilde{\text { irr }^{*}}$ are then defined by

$$
\begin{gathered}
\{F, G\}_{(\tilde{\lambda}, r)} \equiv\left\langle\tilde{\lambda},\left[\frac{\delta F}{\delta \tilde{\lambda}}, \frac{\delta G}{\delta \tilde{\lambda}}\right]\right\rangle_{v}+r \tilde{c}_{v}\left(\frac{\delta F}{\delta \tilde{\lambda}}, \frac{\delta G}{\delta \tilde{\lambda}}\right), \\
F, G \in \tilde{\mathscr{F}}\left(\widetilde{\operatorname{Vir}^{*}}\right), \quad(\tilde{\lambda}, r) \in \widetilde{\operatorname{Vir}}^{*} .
\end{gathered}
$$

Theorem 6.2. The map

$$
\widetilde{J}^{\rho, \mathscr{S}}: \widetilde{L g}^{\wedge} * \rightarrow \widetilde{\operatorname{Vir}^{*}}
$$

defined by

$$
\tilde{J}^{\rho, \mathscr{S}}(\tilde{\mu}, r) \equiv\left(\tilde{J}^{\mathscr{S}}(\tilde{\mu}, r)+i b\left(\dot{\rho}, \tilde{\mu}^{\prime}\right),-r \dot{\rho}^{2}\right)
$$

preserves the Poisson brackets (6.11) and (4.34); i.e., defining

$$
\begin{aligned}
\tilde{J}_{(\tilde{a}, s)}^{\rho, \mathscr{S}}(\tilde{\mu}, r) & \equiv\left\langle\tilde{J}^{\rho, \mathscr{S}}(\tilde{\mu}, r),(\tilde{a}, s)\right\rangle_{v} \\
& =\tilde{J}_{\tilde{a}}^{\mathscr{S}}+i\left\langle b\left(\dot{\rho}, \tilde{\mu}^{\prime}\right), \tilde{a}\right\rangle_{v}-r s \dot{\rho}^{2},
\end{aligned}
$$

we have

$$
\left\{\tilde{\boldsymbol{J}}_{(\tilde{a}, r)}^{\rho, \mathscr{S}}, \tilde{J}_{(\tilde{b}, s)}^{\rho, \mathscr{S}}\right\}=\tilde{\boldsymbol{J}}_{\left([\tilde{a}, \tilde{b}], \tilde{c}_{v}(\tilde{a}, \tilde{b})\right)}^{\rho, \mathscr{S}}
$$

Proof. This follows along the same lines as Theorem 4.4, with the modification

$$
\begin{aligned}
\frac{\delta \tilde{J}_{(\tilde{a}, r)}^{\rho, \mathscr{S}}}{\delta \tilde{\mu}} & =\frac{\delta \tilde{J}_{\tilde{a}}^{\mathscr{S}}}{\delta \tilde{\mu}}-i \dot{\rho} \tilde{a}^{\prime} \\
& =\frac{1}{2 r}\left\{2 \tilde{a} \mathscr{D}(\tilde{\mu})-\mathscr{D}(\tilde{a}) \tilde{\mu}-\frac{\tilde{a}}{r}[\tilde{\mu}, \tilde{\mu}]\right\}-i \dot{\rho} \tilde{a}^{\prime} .
\end{aligned}
$$

In component form, the map $(\widetilde{6.13})$ may be expressed as

$$
\tilde{J}^{\rho, \mathscr{S}}(\tilde{\mu}, r)=\left(\alpha+\theta u,-r \dot{\rho}^{2}\right)
$$

where

$$
\begin{aligned}
& u=\frac{1}{2 r}\left[b(m, m)+b\left(\mu^{\prime}, \mu\right)-\frac{1}{r} b(m,[\mu, \mu])\right]+i b\left(\dot{\rho}, m^{\prime}\right), \\
& \alpha=\frac{1}{2 r}\left[b(m, \mu)-\frac{1}{3 r} b(\mu,[\mu, \mu])\right]+i b\left(\dot{\rho}, \mu^{\prime}\right)
\end{aligned}
$$


and

$$
\tilde{\mu} \equiv \mu+\theta m \text {. }
$$

Returning to the moment map $\tilde{J}^{\rho}$ we may, as in the nontwisted case, develop the components

$$
\tilde{\boldsymbol{J}}^{\rho} \equiv \Gamma^{\rho}+\theta L^{\rho}
$$

in Fourier series:

where

$$
\begin{aligned}
\Gamma^{\rho} & =\sum_{p} \Gamma_{p}^{\rho} e^{-i p \sigma}, \\
L^{\rho} & =\sum_{n \in \mathbb{Z}} L_{n}^{\rho} e^{-i n \sigma},
\end{aligned}
$$

$$
\begin{aligned}
\Gamma_{p}^{\rho} & =\Gamma_{p}-p \sum_{a} \rho_{a} \psi_{a \mid p}, \\
L_{n}^{\rho} & =L_{n}-n \sum_{a} \rho_{a} E_{a \mid n},
\end{aligned}
$$

and

$$
\rho_{a} \equiv b\left(\dot{\rho}, T_{a}\right)
$$

The Poisson bracket relations (6.7) then give:

$$
\begin{aligned}
& \left\{L_{n}^{\rho}, L_{m}^{\rho}\right\}=-i(n-m) L_{n+m}^{\rho}+i k \dot{\rho}^{2} n^{3} \delta_{n+m, 0} \\
& \left\{L_{n}^{\rho}, \Gamma_{p}^{\rho}\right\}=-i\left(\frac{n}{2}-p\right) \Gamma_{n+p}^{\rho}, \\
& \left\{\Gamma_{p}^{\rho}, \Gamma_{q}^{\rho}\right\}=\frac{1}{2} L_{p+q}^{\rho}+k \dot{\rho}^{2} p^{2} \delta_{p+q, 0} .
\end{aligned}
$$

\section{Super Integrable Systems}

In previous work on integrable systems of PDE's, the Miura map, relating the $\mathrm{KdV}$ and MKdV hierarchies, was interpreted as a special case of the "twisted" Sugawara formula and generalized to the non-abelian case [Ku1]. The super KdV system introduced in [Ku2] and the supersymmetric extension of the $\mathrm{KdV}$ system ([MR], [Ma]) may similarly be viewed as super Hamiltonian systems in Vir* and used to define commuting superspace extensions of the MKdV system hierarchies. In this section, we show how the twisted super Sugawara map $(\widetilde{6.13})$ may be used to determine two infinite families of commuting super integrable systems on the super loop space $\widetilde{\mathrm{Lg}}^{\wedge} *$ associated with an arbitrary Lie algebra $\mathfrak{g}$.

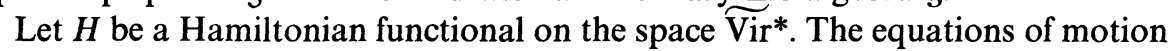
following from the Poisson bracket (6.12) are then:

$$
\begin{aligned}
& u_{t}=\left(u \partial+\partial u+s \dot{\rho}^{2} \partial^{3}\right)\left(\frac{\delta H}{\delta u}\right)+\left(\alpha \partial+\frac{1}{2} \partial \alpha\right)\left(\frac{\delta H}{\delta u}\right), \\
& \alpha_{t}=\left(\partial \alpha+\frac{1}{2} \alpha \partial\right) \frac{\delta H}{\delta u}-\left(\frac{1}{2} u+s \dot{\rho}^{2} \partial^{3}\right)\left(\frac{\delta H}{\delta \alpha}\right), \\
& s_{t}=0
\end{aligned}
$$


where

$$
\tilde{\lambda}(t)=(\alpha(t)+\theta u(t), s)
$$

denotes an integral curve. Define a 1-parameter family of Hamiltonians

$$
H_{c} \equiv \int_{0}^{2 \pi} d \sigma\left[u^{2}-c \alpha \alpha^{\prime}\right]
$$

It is known that for $c=1$ or 2 these define completely integrable extensions of the $\mathrm{KdV}$ system that belong to two hierarchies of commutative flows. The case $c=2$ (super $\mathrm{KdV}$ ) was studied in [Ku2] and $c=1$ (supersymmetric KdV) in [MR], [Ma]. Only the second, which is supersymmetric, admits a superspace formulation, with Hamiltonian $H_{1}$ expressible in terms of Berezinian integration as

$$
H_{1}=\int_{0}^{2 \pi} d \sigma \int d \theta \tilde{\lambda} \mathscr{D}(\tilde{\lambda})
$$

and equations of motion:

$$
\tilde{\lambda}_{t}=3[\tilde{\lambda} \mathscr{D}(\tilde{\lambda})]^{\prime}-\tilde{\lambda}^{\prime \prime \prime} .
$$

In component form, the equations of motion $(7.1 \mathrm{a}-\mathrm{c})$ are

$$
\begin{aligned}
& u_{t}=\left(3 u^{2}-u^{\prime \prime}-3 c \alpha \alpha^{\prime}\right)^{\prime}, \\
& \alpha_{t}=3 u^{\prime} \alpha+(2+c) u \alpha^{\prime}-c \alpha^{\prime \prime \prime},
\end{aligned}
$$

where we have fixed the level set of the trivial invariant to be

$$
s=\frac{1}{2}
$$

to obtain the standard normalizations.

Now, denote by

$$
H^{*} \equiv H \circ \tilde{\boldsymbol{J}}^{\rho, \mathscr{S}} \in \tilde{\mathscr{F}}\left(\widetilde{L g}^{\wedge}{ }^{*}\right)
$$

the pull-back of $H \in \tilde{\mathscr{F}}\left(\widetilde{\operatorname{Vir}^{*}}\right)$ to $\widetilde{\mathrm{Lg}^{\wedge}}$. The equations of motion in $\widetilde{\mathrm{Lg}}^{\wedge}$ for Hamiltonian $H^{*}$ and Poisson bracket (4.34) are

$$
\begin{aligned}
& m_{t}=-\left[m, \frac{\delta H^{*}}{\delta m}\right]+r \partial\left(\frac{\delta H^{*}}{\delta m}\right)-\left[\mu, \frac{\delta H^{*}}{\delta \mu}\right] \\
& \mu_{t}=-\left[\mu, \frac{\delta H^{*}}{\delta m}\right]-r \frac{\delta H^{*}}{\delta \mu} \\
& r_{t}=0
\end{aligned}
$$

where

$$
(\tilde{\mu}(t), r(t))=(\mu(t)+\theta m(t), r(t))
$$

denotes an integral curve. The canonical map $(\widetilde{6.13})$, defined in components by (6.13), (6.16) and (6.17), takes such curves into solutions of $(7.1 \mathrm{a}-\mathrm{c})$.

For the case $H_{c}$ of Eq. (7.3), we have 


$$
\begin{aligned}
& \frac{\delta H_{c}^{*}}{\delta m}=\frac{2}{r} u m+2 i u^{\prime} \dot{\rho}-\frac{u}{r^{2}}[\mu, \mu]-\frac{c}{r} \mu^{\prime} \alpha, \\
& \frac{\delta H_{c}^{*}}{\delta \mu}=-\frac{1}{r}\left(\mu u^{\prime}+2 \mu^{\prime} u\right)+2 \frac{u}{r^{2}}[m, \mu]+2 i c \alpha^{\prime \prime} \dot{\rho}-\frac{c}{r} m \alpha^{\prime}+\frac{c}{r^{2}} \alpha^{\prime}[\mu, \mu],
\end{aligned}
$$

where

$$
r \dot{\rho}^{2}=-\frac{1}{2}
$$

for the level set (7.7), with $u$ and $\alpha$ defined by Eqs. (6.16), (6.17). Substituting $(7.11 \mathrm{a}-\mathrm{b}),(7.12)$ in $(7.9 \mathrm{a}-\mathrm{c})$, we get nonabelian generalizations of the super MKdV $(c=2)$ and supersymmetric $\operatorname{MKdV}(c=1)$ systems. To recover the purely bosonic systems, set

$$
\mu=0, \quad \alpha=0 .
$$

Then Eq. (6.16) becomes

$$
u=\frac{1}{2 r}\left[b(m, m)+2 \operatorname{irb}\left(\dot{\rho}, m^{\prime}\right)\right]
$$

and (7.9a) becomes

$$
\begin{aligned}
m_{t}= & \frac{1}{r^{2}}\left[b(m, m)+2 i r b\left(\dot{\rho}, m^{\prime}\right) m-i\left(r b(m, m)+2 i r^{2} b\left(\dot{\rho}, m^{\prime}\right)\right) \dot{\rho}\right]^{\prime} \\
& +\frac{i}{r}\left[b(m, m)+2 i r b\left(\dot{\rho}, m^{\prime}\right)\right]^{\prime}[m, \dot{\rho}] .
\end{aligned}
$$

In particular, if $m=v$ is a scalar $\left(\mathfrak{g}=\mathbb{R}^{1}\right)$, this becomes

$$
v_{t}=\left(\frac{v^{3}}{r^{2}}-r v^{\prime \prime}\right)^{\prime}
$$

the usual MKdV equation.

By applying the same procedure of pulling back Hamiltonians on $\widetilde{\text { Vir* }}$ under the map (6.13) to the full commuting hierarchies of super-KdV ([Ku2]) and supersymmetric $\mathrm{KdV}$ ([MR], [Ma]) type, we obtain two infinite hierarchies of commuting flows in the dual $\widehat{\mathrm{Lg}^{\wedge}}$ of any super loop algebra with Ad-invariant metric. These then provide super extensions of the corresponding generalized MKdV hierarchies.

\section{Quantization and Representation Theory}

The irreducible unitary highest weight representations of $\widetilde{\mathrm{Vir}} \ltimes \widetilde{\mathrm{Lg}^{\wedge}}$ for compact $G$ were constructed by Kac and Todorov in [KT]. In this section, we briefly indicate how quantization of the Poisson bracket algebra defined by Eqs. (5.7), (5.11) and (5.12), together with the classical Sugawara formula (5.10), leads to the results of $[\mathrm{KT}]$.

First, since the super phase space $\widetilde{L G} / G$ may, according to the discussion 
following Theorem 3.4, be viewed as a product of the bosonic phase space $L G / G$ and the fermionic one $L_{\mathfrak{g}}^{ \pm}$, quantization leads to the tensor product

$$
\mathscr{H}=\mathscr{H}_{F} \otimes \mathscr{H}_{B}
$$

of a Fermionic Hilbert space $\mathscr{H}_{F}$ with a bosonic one $\mathscr{H}_{B}$. The quiantized operators corresponding to the classical generators $\left\{\psi_{a \mid p}, E_{a \mid n}^{F}, E_{a \mid n}^{B}, E_{a \mid n}, \Gamma_{p}, L_{n}\right\}$ will be denoted $\left\{\hat{\psi}_{a \mid p}, \hat{E}_{a \mid n}^{F}, \hat{E}_{a \mid n}^{B}, \hat{E}_{a \mid n}, \hat{\Gamma}_{p}, \hat{L}_{n}\right\}$. From the Clifford algebra defined by the Poisson brackets (5.7a), standard quantization of free Fermions leads to the fermionic Fock space $\mathscr{H}_{F}$ as an infinite wedge product space (i.e. the infinite dimensional irreducible Clifford module), with positive Fermi components $\left\{\hat{\psi}_{a \mid p}\right\}_{p>0}$ acting as annihilation operators and negative ones $\left\{\hat{\psi}_{a \mid p}\right\}_{p<0}$ as creation operators. For Ramond boundary conditions, the operators $\left\{\hat{\psi}_{a \mid 0}\right\}$ are represented, as usual (see, e.g. [GO], Sect. 5.1), by Dirac matrices on the $2^{[d / 2]}$-dimensional Clifford module of vacuum vectors $(d \equiv \operatorname{dim} g)$. The quantization $E_{a \mid n}^{B} \rightarrow \hat{E}_{a \mid n}^{B}$ may be done in a variety of ways (see, e.g. [PS], [W], [H]), each leading to irreducible highest weight representations of the affine $\mathrm{Kac}-$ Moody algebra $\mathrm{Lg}^{\wedge}$ on a bosonic Fock space $\mathscr{H}_{B}$. The classical relation (5.6) may be quantized to define a representation of $L \mathfrak{g}^{\wedge}$ on $\mathscr{H}_{F}$ generated by the operators

$$
\hat{E}_{a \mid n}^{F}=\frac{1}{2 k} \sum_{p} \sum_{b, c} f_{a b}^{c}: \psi_{b \mid p} \psi_{c \mid n-p}: .
$$

The meaning of the normal ordering operation : $\cdots:$ is, as usual, that annihilation operators are placed to the right, (cf. [GO], Sect. (5.1)).

For purposes of comparison with $[\mathrm{KT}]$, we give the following table of notational equivalents.

Table I.

\begin{tabular}{ll}
\hline Present Notation & Notation of $[\mathrm{KT}]$ \\
\hline$\hat{\psi}_{a \mid p}$ & $e^{i(\pi / 4)} h_{p}^{a}=e^{i(\pi / 4)} h_{p}^{a} \sqrt{\frac{\lambda(q)+c_{2}}{c_{2}}}$ \\
$\hat{E}_{a \mid n}^{F}$ & $Q_{n}^{a}$ \\
$\hat{E}_{\mid n n}^{B}$ & $q_{n}^{a}$ \\
$\hat{E}_{a \mid n}$ & $Q_{n}^{a}$ \\
$\hat{\Gamma}_{p}$ & $e^{i(\pi / 4)} \frac{G_{p}}{2}$ \\
$\hat{L}_{n}$ & $L_{n}$ \\
$k=k_{B}+\frac{c_{2}}{2}$ & $\frac{\lambda}{2}=\frac{\lambda(q)+c_{2}}{2}$ \\
& \\
\hline
\end{tabular}

The quantity $c_{2}$ is the eigenvalue of the quadratic Casimir operator for $g$ in the adjoint representation. Equation (8.2) corresponds to Eq. (4.2) of [KT] (see also [GO], Sect. 5.2). The operators $\left\{\hat{\psi}_{a \mid p}, \hat{E}_{a \mid n}^{F}, \hat{E}_{a \mid n}^{B}\right\}$ are extended trivially to the tensor product $\mathscr{H}_{F} \otimes \mathscr{H}_{B}$. 
The operators $\left\{\hat{\psi}_{a \mid p}, \hat{E}_{a \mid n}^{F}\right\}$, with

$$
\widehat{E}_{a \mid n} \equiv \hat{E}_{a \mid n}^{F}+\hat{E}_{a \mid n}^{B}
$$

then define an irreducible unitary highest weight representation of $\widetilde{\mathrm{Lg}^{\wedge}}$, provided the original representation of $L g^{\wedge}$ on $\mathscr{H}_{B}$ was such. The quantized version of $(5.7 \mathrm{a}-\mathrm{f})$ becomes

$$
\begin{aligned}
{\left[\hat{\psi}_{a \mid p}, \hat{\psi}_{b \mid q}\right]_{+} } & =i k \delta_{a b} \delta_{p+q, 0}, \\
{\left[\hat{E}_{a \mid n}, \hat{\psi}_{b \mid q}\right] } & =i \sum_{c} f_{a b}^{c} \hat{\psi}_{c \mid n+q}, \\
{\left[\hat{E}_{a \mid n}, \hat{E}_{b \mid m}\right] } & =i \sum_{c} f_{a b}^{c} \hat{E}_{c \mid n+m}+n k \delta_{n+m, 0} \delta_{a b}, \\
{\left[\hat{E}_{a \mid n}^{B}, \hat{E}_{b \mid m}^{B}\right] } & =i \sum_{c} f_{a b}^{c} \hat{E}_{c \mid n+m}^{B}+n k_{B} \delta_{n+m, 0} \delta_{a b}, \\
{\left[\hat{E}_{a \mid n}^{F}, \hat{\psi}_{b \mid q}\right] } & =i \sum_{c} f_{a b}^{c} \hat{\psi}_{c \mid n+q}, \\
{\left[\hat{E}_{a \mid n}^{F}, \hat{E}_{b \mid m}^{F}\right] } & =i \sum_{c} f_{a b}^{c} \hat{E}_{c \mid n+m}^{F}+n \frac{c_{2}}{2} \delta_{n+m, 0} \delta_{a b} .
\end{aligned}
$$

The value of the central term $k$ is, as given in Table 1 ([KT], Theorem 4),

$$
k=k_{B}+\frac{c_{2}}{2},
$$

where $k_{B}(\equiv \lambda(q) / 2)$ denotes the corresponding value for the bosonic affine KacMoody representation generated by $\left\{\hat{E}_{a \mid n}^{B}\right\}$.

The extension of these representations to $\widetilde{\mathrm{Vir}} \ltimes \widetilde{\mathrm{Lg}}^{\wedge}$ involves additional normal ordering in the quantized form of the super Sugawara formulas $(5.9 a, b)$, namely

$$
\begin{aligned}
& \hat{\Gamma}_{p}=\frac{1}{2 k} \sum_{n} \sum_{a}:\left[\hat{E}_{a \mid n}^{B} \hat{\psi}_{a \mid p-n}+\frac{1}{3} \hat{E}_{a \mid n}^{F} \hat{\psi}_{a \mid p-n}\right]: \\
& \hat{L}_{n}=\frac{1}{2 k}\left\{\sum_{m} \sum_{a}: \hat{E}_{a \mid m}^{B} \hat{E}_{a \mid n-m}^{B}:-\sum_{p} \sum_{a} i p: \hat{\psi}_{a \mid p} \hat{\psi}_{a \mid n-p}:\right\} .
\end{aligned}
$$

The normal ordering in the first term of (8.6a) is irrelevant, since the factors commute, while the second is ordered as in [KT], Eq. (5.6). The formula (8.6b) is not explicitly given in [KT], but the normal ordering for the bosonic part is just the usual one for the bosonic Sugawara formula, while that for the fermionic part is the same as in the free quark model ([GO], Sects. 4.1 and 5.4).

The quantized form of the Poisson bracket relations $(5.12 \mathrm{a}-\mathrm{d})$ becomes:

$$
\begin{aligned}
& {\left[\hat{L}_{n}, \hat{E}_{a \mid m}\right]=-m \hat{E}_{a \mid n+m},} \\
& {\left[\hat{L}_{n}, \hat{\psi}_{a \mid p}\right]=-\left(p+\frac{n}{2}\right) \hat{\psi}_{a \mid n+p},} \\
& {\left[\hat{\Gamma}_{p}, \hat{E}_{a \mid n}\right]=-\frac{n}{2} \hat{\psi}_{a \mid n+p},}
\end{aligned}
$$




$$
\left[\hat{\Gamma}_{p}, \hat{\psi}_{a \mid q}\right]_{+}=\frac{i}{2} \hat{E}_{a \mid p+q}
$$

Finally, the quantized version of $(5.11 \mathrm{a}-\mathrm{c})$ gives a representation of the super Virasoro algebra:

$$
\begin{aligned}
{\left[\hat{L}_{n}, \hat{L}_{m}\right] } & =(n-m) \hat{L}_{n+m}+\frac{c}{12} n\left(n^{2}-1\right) \delta_{n+m, 0} \\
{\left[\hat{L}_{n}, \hat{\Gamma}_{p}\right] } & =\left(\frac{n}{2}-p\right) \hat{\Gamma}_{n+p} \\
{\left[\hat{\Gamma}_{p}, \hat{\Gamma}_{q}\right]_{+} } & =\frac{i}{2} \hat{L}_{p+q}+i \frac{c}{12}\left(p^{2}-\frac{1}{4}\right) \delta_{p+q, 0}
\end{aligned}
$$

where, according to [KT], Theorem 4, the value of the central charge $c$ is:

$$
c=\frac{d}{2}\left(1+\frac{k_{B}}{k}\right) .
$$

If the same quantization procedure is applied to the "twisted" generators of Sect. 6 , we obtain the operators

$$
\begin{gathered}
\hat{\Gamma}_{p}^{\rho}=\hat{\Gamma}_{p}-p \sum_{a} \rho_{a} \hat{\psi}_{a \mid p}, \\
\hat{L}_{n}^{\rho}=\hat{L}_{n}-n \sum_{a} \rho_{a} \hat{E}_{a \mid n} .
\end{gathered}
$$

The resulting central charge is the sum of that obtained classically, as in Eqs. $(6.23 \mathrm{a}-\mathrm{c})$, plus that obtained from the quantization:

$$
\begin{aligned}
{\left[\hat{L}_{n}^{\rho}, \hat{L}_{m}^{\rho}\right] } & =(n-m) \hat{L}_{n+m}^{\rho}+\left[\frac{c}{12} n\left(n^{2}-1\right)-k \dot{\rho}^{2} n^{3}\right] \delta_{n+m, 0} \\
{\left[\hat{L}_{n}^{\rho}, \hat{\Gamma}_{p}^{\rho}\right] } & =\left(\frac{n}{2}-p\right) \hat{\Gamma}_{n+m}^{\rho}, \\
{\left[\hat{\Gamma}_{p}^{\rho}, \hat{\Gamma}_{q}^{\rho}\right]_{+} } & =\frac{i}{2} \hat{L}_{p+q}^{\rho}+i \frac{c}{12}\left(p^{2}-\frac{1}{4}\right)+i k \dot{\rho}^{2} p^{2} \delta_{p+q, 0} .
\end{aligned}
$$

\section{References}

[GO] Goddard, P., Olive, D.: Kac-Moody and Virasoro algebras in relation to quantum physics. Int. J. Mod. Phys. A1, 303 (1986)

[GSW] Green, M. B., Schwarz, J. H., Witten, E.: Superstring Theory, Vol. I. Cambridge, New York: Cambridge University Press 1987

[H] Harnad, J.: Constrained Hamiltonian Systems on Lie Groups, Moment Map Reductions and Central Extensions, (preprint, CRM 1989)

[HK] Harnad, J., Kupershmidt, B. A.: Twisted Diff $S^{1}$-action on loop groups and representations of the Virasoro algebra. Lett. Math. Phys. 19, 277-284 (1990)

[K] Kostant, B.: Graded manifolds, graded Lie theory and prequantization. In: Differential Geometric Methods in Physics. Bleuler, K., Reetz, A. (eds). Lecture Notes in Mathematics, vol. 570, pp. 177-306. Berlin, Heidelberg, New York: Springer 1977

[KT] Kac, V. G., Todorov, I. T.: Superconformal current algebras and their unitary representations. Commun. Math. Phys. 102, 337-347 (1985) 
[Ku1] Kupershmidt, B.: Modified Korteweg-de Vries equations on Euclidean Lie algebras. Int. J. Mod. Phys. (to appear, 1989)

[Ku2] Kupershmidt, B. A.: A super Korteweg-de Vries equation: an integrable system. Phys. Lett. A102, 213 (1984)

[M] Miura, R. M.: Korteweg-de Vries equation and generalizations I. A remarkable explicit nonlinear transformation. J. Math. Phys. 9, 1202-1204 (1968)

[Ma] Mathieu, P.: Supersymmetric extension of the Korteweg-de Vries equation. J. Math. Phys. 29, 2499-2506 (1988)

[MR] Manin, Yu. I., Radul, A. O.: A supersymmetric extension of the Kadomtsev-Petviashvili hierarchy. Commun. Math. Phys. 98, 65-77 (1985)

[PS] Pressley, A., Segal, G.: Loop Groups. Oxford: Clarendon Press 1986

[S] Sugawara, H.: A field theory of currents. Phys. Rev. 176, 2019-2025 (1968)

[W] Witten, E.: Non-abelian bosonization in two dimensions. Commun. Math. Phys. 92, 454-472 (1984)

Communicated by A. Jaffe

Received August 21, 1989 
